\title{
EFFECTIVE MULTIPLICITY FOR THE EINSTEIN-SCALAR FIELD LICHNEROWICZ EQUATION
}

\author{
BRUNO PREMOSELLI
}

\begin{abstract}
We prove the stability of the Einstein-scalar field Lichnerowicz equation under subcritical perturbations of the critical nonlinearity in dimensions $n=3,4,5$. As a consequence, we obtain the existence of a second solution to the equation in several cases. In particular, in the positive case, including the CMC positive cosmological constant case, we show that each time a solution exists, the equation produces a second solution with the exception of one critical value for which the solution is unique.
\end{abstract}

\section{Statement of the Results.}

Let $(M, g)$ be a smooth closed Riemannian manifold of dimension $n \geq 3$. We are interested in the Einstein-scalar field Lichnerowicz equation in $M$ :

$$
\triangle_{g} u+h u=f u^{2^{*}-1}+\frac{a}{u^{2^{*}+1}},
$$

where $h, f$ and $a$ are smooth functions on $M, \triangle_{g}=-\operatorname{div}_{g} \nabla$ is the Laplace-Beltrami operator, $2^{\star}=\frac{2 n}{n-2}$ is the critical Sobolev exponent for the Sobolev space $H^{1}$, and we assume that $\triangle_{g}+h$ is coercive, $a \geq 0, a \not \equiv 0$, and $\max _{M} f>0$. By closed, following standard terminology, we mean compact without boundary.

Equation (EL) arises in the mathematical analysis of general relativity when solving the Einstein equations in a scalar-field setting, when the gravity is coupled to a scalar-field $\psi$. Special important cases include the massive Klein-Gordon setting or the case of a positive cosmological constant $\Lambda$. Given a closed manifold $(M, g)$ of dimension $n \geq 3$ endowed with two smooth functions $\pi$ and $\psi$ and a (2,0)-symmetric tensor field $K$, the Cauchy problem in general relativity consists in finding a Lorentzian manifold $(M \times \mathbb{R}, \tilde{g})$ together with a smooth function $\tilde{\psi}$ on $M \times \mathbb{R}$ such that $\tilde{\psi}_{\mid M}=\psi$ and $\partial_{n} \tilde{\psi}_{\mid M}=\pi$, where $\partial_{n}$ denotes the normalized time derivative, such that $K$ is the second fundamental form of the embedding $M \subset M \times \mathbb{R}$, and such that $(M \times \mathbb{R}, \tilde{g})$ satisfies the Einstein equations:

$$
\operatorname{Ric}(\tilde{g})_{a b}+\frac{1}{2} R(\tilde{g}) \tilde{g}_{a b}=T_{a b},
$$

where $\operatorname{Ric}(\tilde{g})$ is the Ricci tensor of $\tilde{g}, R(\tilde{g})$ is its scalar curvature and $T$ is the stress-energy tensor-field. This tensor field depends on $\tilde{g}$, on $\tilde{\psi}$ and on some potential $V$, itself related to $\tilde{\psi}$ by some wave equation. As shown first by Choquet-Bruhat [1] for the vacuum case, see also Choquet-Bruhat-Isenberg-Pollack [6], a necessary and sufficient condition for the existence of such a $\tilde{g}$ on $M \times \mathbb{R}$ is that the following system of equations in $M$ is satisfied:

$$
\begin{cases}R(g)+\operatorname{tr}_{g} K^{2}-\|K\|_{g}^{2} & =\pi^{2}+|\nabla \psi|_{g}^{2}+2 V(\psi) \\ \partial_{i}\left(\operatorname{tr}_{g} K\right)-K_{i, j}^{j} & =\pi \partial_{i} \psi,\end{cases}
$$

where $R(g)$ is the scalar curvature of $g$ and $\nabla$ refers to the Levi-Civita connection of $g$. By specifying some of the unknown initial data $(g, K, \psi, \pi)$ and solving the system for the remaining data, the conformal method initiated by Lichnerowicz [17] allows to turn (1.1) into a system of 
elliptic partial differential equations of critical Sobolev growth, called the conformal constraint system of equations. For a survey reference on the constraint equations see Bartnik-Isenberg 3 and for further informations on the conformal method see Choquet-Bruhat, Isenberg and Pollack [7. Essentially, the set of free data consists of $(\psi, \tau, \pi, U)$, where $\psi, \tau, \pi$ are smooth functions in $M$ and $U$ is a smooth symmetric traceless and divergence-free $(2,0)$-tensor in $M$. Given $(\psi, \tau, \pi, U)$ an initial free data set, the conformal constraint system of equations, whose unknowns are a smooth positive function $\varphi$ in $M$ and a smooth vector field $W$ in $M$, is written as

$$
\begin{cases}\triangle_{g} \varphi+\mathcal{R}_{\psi} \varphi & =\mathcal{B}_{\tau, \psi, V} \varphi^{2^{*}-1}+\frac{\mathcal{A}_{\pi, U}(W)}{\varphi^{2^{*}+1}} \\ \triangle_{g, \text { conf }} W & =\frac{n-1}{n} \varphi^{2^{*}} \nabla \tau-\pi \nabla \psi\end{cases}
$$

where we have let:

$$
\begin{aligned}
& \mathcal{R}_{\psi}=c_{n}\left(R(g)-|\nabla \psi|_{g}^{2}\right), \\
& \mathcal{B}_{\tau, \psi, V}=c_{n}\left(2 V(\psi)-\frac{n-1}{n} \tau^{2}\right), \\
& \mathcal{A}_{\pi, U}(W)=c_{n}\left(\left|U+\mathcal{L}_{g} W\right|_{g}^{2}+\pi^{2}\right),
\end{aligned}
$$

and $c_{n}=\frac{n-2}{4(n-1)}$. In the above $\triangle_{g, \text { conf }} W=\operatorname{div}_{g}\left(\mathcal{L}_{g} W\right)$ and $\mathcal{L}_{g} W$ is the symmetric trace-free part of $\nabla W$ given by $\mathcal{L}_{g} W_{i j}=W_{i, j}+W_{j, i}-\frac{2}{n} \operatorname{div}_{g} W g_{i j}$. Vector fields satisfying $\mathcal{L}_{g} W=0$ are called conformal Killing vector fields. In the CMC case, which by definition corresponds to $\nabla \tau \equiv 0$, the system (1.2) is semi-decoupled. The second equation in (1.2) has a unique solution $W$ when $g$ has no conformal Killing vector fields. The first equation in (1.2) is then nothing but (EL) with

$$
h=\mathcal{R}_{\psi}, \quad f=\mathcal{B}_{\tau, \psi, V}, \text { and } a=\mathcal{A}_{\pi, U}(W) .
$$

Note that conformal Killing vector fields generically do not exist by Beig, Chruściel and Schoen 4]. Several existence results for (EL) have been obtained. When $f \leq 0$ the equation is fully understood, see Isenberg [15] or Choquet-Bruhat, Isenberg and Pollack [7]. Partial existence results are known when $\max _{M} f>0$, see Hebey, Pacard and Pollack [12] and Ngô and Xu [20]. The main result of the paper is as follows.

Theorem 1.1. Let $(M, g)$ be a n-dimensional closed Riemannian manifold with $3 \leq n \leq 5$ and $h, f$ and $a$ be smooth functions on $M$. Assume that $\triangle_{g}+h$ is coercive, $a \geq 0$, $a \neq \equiv$ and $\max _{M} f>0$. Consider the following Einstein-Lichnerowicz equation in $M$, for $\theta>0$ :

$$
\triangle_{g} u+h u=f u^{2^{*}-1}+\frac{\theta a}{u^{2^{*}+1}} .
$$

Then there exist $0<\theta_{1} \leq \theta_{2} \leq+\infty$ such that equation EL $E$ has:

- at least two solutions if $\theta<\theta_{1}$,

- no solutions for $\theta>\theta_{2}$,

- at least one solution if $\theta_{1} \leq \theta<\theta_{2}$.

In case $f>0, \theta_{2}$ is finite, there holds $\theta_{1}=\theta_{2}$, and $E L_{\theta}$ has one and only solution for $\theta=\theta_{1}=\theta_{2}$. In particular, when $f>0$ in $M$, there exists $\theta_{\star} \in(0,+\infty)$ such that EL has at least two solutions if $\theta<\theta_{\star}$, exactly one solution if $\theta=\theta_{\star}$, and no solution if $\theta>\theta_{\star}$.

A few multiplicity results concerning (EL) were known. Ma and Wei [18] showed the existence of two smooth positive solutions of $(E L)$ in dimensions $3 \leq n \leq 5$ when $h, f$ and $a$ are positive assuming the strict stability of the minimal solution but without investigating its stability. In another context, Ngô-Xu 20] showed the existence of a second solution of EL if the first eigenvalue of the operator $\triangle_{g}+h$ is negative. Assuming that $\triangle_{g}+h$ is coercive, no results were known under the sole assumption $\max _{M} f>0$ that we use in Theorem 1.1. Assuming that $f$ is 
positive, Theorem 1.1 shows that each time a solution exists, there is at least another solution to the equation, except in the limit-case $\theta=\theta_{\star}$. In particular, the $f>0$ case in Theorem 1.1 is proved by showing that the minimal solution of $(E L)$ is always strictly stable, except in the limitcase $\theta=\theta_{\star}$, where it always fails to be strictly stable. The uniqueness in the limit case $\theta=\theta_{\star}$ is then obtained as a consequence of its non-strict stability. This of course highly complements the Ma and Wei [18] result and shows that the Ma-Wei [18] alternative only occurs in the limit case $\theta=\theta_{\star}$. Needless to say, Theorem 1.1 can be applied to the conformal constraint system (1.2) in the CMC case. As a direct consequence of our theorem, noting that $\mathcal{A}_{\theta \pi, \theta U}(W)=\theta^{2} \mathcal{A}_{\pi, U}(W)$ in the CMC-case, we get that the following corollary holds true. The corollary includes, as a special case, the CMC positive cosmological constant case.

Corollary 1.2. Let $(M, g)$ be a closed Riemannian manifold of dimension $3 \leq n \leq 5$ of positive Yamabe type such that $g$ has no conformal Killing vector fields. Let $V$ be a smooth positive function on $\mathbb{R}$, and let $\psi$ be a smooth function in $M$ such that the operator $\triangle_{g}+\mathcal{R}_{\psi}$ is coercive. Assume that $(\tau, \pi, U)$ satisfies that $\tau \equiv C^{\text {st }}$ (CMC case), $(\pi, U) \not \equiv(0,0)$, and

$$
\frac{n-1}{n} \tau^{2}<2 \min _{x \in M} V(\psi(x)) .
$$

Then there exists $\theta_{\star} \in(0,+\infty)$ such that the conformal constraint system

$$
\begin{cases}\triangle_{g} \varphi+\mathcal{R}_{\psi} \varphi & =\mathcal{B}_{\tau, \psi, V} \varphi^{2^{*}-1}+\frac{\mathcal{A}_{\theta \pi, \theta U}(W)}{\varphi^{*}+1} \\ \triangle_{g, \text { conf }} W & =\frac{n-1}{n} \varphi^{2^{*}} \nabla \tau-\pi \nabla \psi\end{cases}
$$

has at least two solutions if $\theta<\theta_{\star}$, exactly one solution if $\theta=\theta_{\star}$, and no solution if $\theta>\theta_{\star}$.

Proof. Since $\mathcal{A}_{\theta \pi, \theta U}(W)=\theta^{2} \mathcal{A}_{\pi, U}(W)$ is nonnegative we first need to show that $\mathcal{A}_{\pi, U}(W)$ is non zero, where $W$ is the unique solution of the vector equation in (1.5) when $\nabla \tau=0$. Using equation (1.3), this is automatically true if $\pi \neq \equiv 0$. If $\pi \equiv 0$ since $g$ has no conformal Killing fields this implies $W=0$ and hence $U+\mathcal{L}_{g} W$ is not identically zero as soon as $U$ is not everywhere zero. Corollary 1.2 then easily follows from Theorem 1.1 since (1.4) implies that $\mathcal{B}_{\tau, \psi, V}>0$.

The proof of Theorem 1.1 goes through the proof of an involved stability result for $(E L)$ under subcritical, asymptotically critical perturbations of the nonlinear power, and in particular makes use of blow-up analysis in the Sobolev critical setting. We state our stability result, Theorem 2.1 in section 2, In section 3 we perform an asymptotic analysis of blowing-up sequences of solutions of our asymptotically critical equations to obtain sharp asymptotic estimates. They are used in section 4 to prevent the appearence of concentration points and to prove Theorem 2.1] In section 5 we show that, if $3 \leq n \leq 5$, each time the critical equation $E L$ has a mountain-pass structure it admits at least two smooth positive solutions. This result is obtained through a variational analysis of the subcritical equations obtained from $(E L)$ and Theorem 2.1. Section 6 is devoted to the construction of a minimal solution of $(E L)$ for the $L^{\infty}(M)$-norm. Theorem 1.1 is proved in section 7 , using the results of sections 5 and 6 .

Acknowledgements. The author warmly thanks Olivier Druet and Emmanuel Hebey for constant support and valuable remarks during the elaboration of this paper.

\section{Stability of the EQuation $E L$ )}

We present here the stability result we have for $(E L)$ which we will use to prove Theorem 1.1 Our stability result establishes that, in low dimensions, equation $(E L)$ is stable under sub-critical perturbations of its critical exponent and perturbations of the coefficient $a$. 
Theorem 2.1. Let $(M, g)$ be a $n$-dimensional closed Riemannian manifold with $3 \leq n \leq 5$ and $h, f$ and $a$ be smooth functions on $M$. Assume that $\triangle_{g}+h$ is coercive, a is non-negative, $a \not \equiv 0$ and $\max _{M} f>0$. Let $\left(a_{k}\right)_{k}$ be a sequence of non-negative functions converging in $C^{0}(M)$ to a as $k \rightarrow \infty$ and $\left(q_{k}\right)_{k}, 2 \leq q_{k} \leq 2^{*}$, be a sequence of positive real numbers converging to $2^{*}$. Consider the following (sub) critical perturbations of (EL):

$$
\triangle_{g} u+h u=f u^{q_{k}-1}+\frac{a_{k}}{u^{q_{k}+1}} .
$$

Let $\left(u_{k}\right)_{k}$ be a sequence of solutions of $E L_{k}$ and assume that either $f>0$ or $\left(u_{k}\right)_{k}$ is uniformly bounded in $H^{1}$. Then there exists a smooth positive solution $u$ of (EL) such that, up to a subsequence, $u_{k}$ converges to $u$ in the $C^{1, \alpha}$ topology for any $0<\alpha<1$.

Two stability results related to $(E L)$ exist in the literature. Druet-Hebey proved in [10], in dimension 3 to 5 , the stability and bounded-stability of $E L$ when perturbing the coefficients $h$, $f$ and $a$ without changing the critical exponent. In [10] are also found examples of instability for (EL) when $n \geq 6$. Also Hebey-Veronelli 13 proved the stability of EL in the Einstein-Maxwell theory when $n=3$ and $f=C^{s t}$. The key point in Theorem 2.1 with respect to [10] and [13] is that we allow fully subcritical perturbations of $E L$ which makes the analysis more involved as $q_{k} \rightarrow 2^{*}$ with $q_{k}<2^{*}$ for all $k$. This is crucial in order to obtain the existence of two solutions in Theorem 1.1, which are constructed as limits of solutions of the subcritical equations associated to $(E L)$, see section 5 .

As an interesting remark it can be noted that the generic existence of two solutions in Theorem 1.1 is implicitly contained in Theorem 2.1 through a degree-theory argument. Consider for instance the case $f>0$. We compute the degree of $E L_{\theta}$ mimicking a computation carried out by Schoen [26] for the Yamabe equation. Since $f>0$ both the existence of a solution of $E L_{\theta}$ when $\theta \ll 1$ and the non-existence when $\theta \gg 1$ were proven in Hebey-Pacard-Pollack [12]. Let for any positive $M$

$$
\Omega_{M}=\left\{u \in C^{2}(M) \text { such that }\|u\|_{C^{2}(M)} \leq M \text { and } \inf _{M} u \geq \frac{1}{M}\right\},
$$

and let $\theta^{-}<\theta^{+}$be such that equation $E L_{\theta}$ has at least a solution for $\theta=\theta^{-}$and has none for $\theta=\theta^{+}$. Define, for any $\theta^{-} \leq \theta \leq \theta^{+}, J_{\theta}:\left\{u \in C^{2}(M), \inf _{M} u>0\right\} \rightarrow C^{2}(M)$ by

$$
J_{\theta}(u)=u-\left(\triangle_{g}+h\right)^{-1}\left(f u^{2^{*}-1}+\frac{\theta a}{u^{2^{*}+1}}\right) .
$$

By Theorem 2.1, there exists a positive $M_{0}$ such that $J_{\theta}^{-1}(\{0\}) \subset \Omega_{M_{0}}$ for all $\theta^{-} \leq \theta \leq \theta^{+}$, so that the Leray-Schauder $\operatorname{degree} \operatorname{deg}\left(J_{\theta}, \Omega_{M}, 0\right)$ is well-defined for $\theta^{-} \leq \theta \leq \theta^{+}$and for any $M>M_{0}$. Since it is homotopy-invariant and no solutions of $E L_{\theta}$ exist for $\theta=\theta^{+}$, there holds

$$
\operatorname{deg}\left(J_{\theta}, \Omega_{M}, 0\right)=0
$$

for any $M>M_{0}$ and any $\theta^{-} \leq \theta \leq \theta^{+}$. In particular, 2.1) shows that solutions of $E L_{\theta}$ generically appear by pairs. Theorem 1.1 shows that two solutions actually always exist.

Existence of at least one solution for (1.2) without the CMC-assumption can be found in AllenClausen-Isenberg [1, Dahl-Gicquaud-Humbert [8, Holst-Nagy-Tsogtgerel [14] and Maxwell [19] when $\mathcal{B}_{\tau, \psi, V}<0$, and in Premoselli [21] when $\mathcal{B}_{\tau, \psi, V}>0$.

\section{Sharp BLOW-UP EStimates.}

We let $(M, g)$ be a smooth closed Riemannian manifold of dimension $3 \leq n \leq 5, h, f$ and $a$ be smooth functions on $M$ satisfying the assumptions of Theorem 2.1. We recall that $\triangle_{g}+h$ is 
coercive if there exists a positive constant $C$ such that for any $u \in H^{1}(M)$,

$$
\int_{M}\left(|\nabla u|_{g}^{2}+h u^{2}\right) d v_{g} \geqslant C\|u\|_{H^{1}(M)}^{2}
$$

or, equivalently, if

$$
\|u\|_{H_{h}^{1}}=\left(\int_{M}\left(|\nabla u|_{g}^{2}+h u^{2}\right) d v_{g}\right)^{\frac{1}{2}}
$$

is an equivalent norm on $H^{1}(M)$. In this case we define the constant $S_{h}$ as the smallest positive constant satisfying for all $u \in H^{1}(M)$ :

$$
\|u\|_{L^{2^{*}}} \leq S_{h}^{\frac{1}{2^{*}}}\|u\|_{H_{h}^{1}} .
$$

We let $\left(a_{k}\right)_{k}$ be a sequence of non-negative functions converging to $a$ in $C^{0}(M)$ as $k \rightarrow \infty$ and $\left(q_{k}\right)_{k}, 2 \leq q_{k} \leq 2^{*}$, be a sequence of positive real numbers converging to $2^{*}$. Let $\left(u_{k}\right)_{k}$ be a sequence of solutions of $E L_{k}$. We assume that the following assumption holds:

$$
\text { either } f>0 \text { or }\left\|u_{k}\right\|_{H^{1}} \leq C_{0} \text {, }
$$

where $C_{0}$ is a positive constant independent of $k$. As a first striking fact, we prove that the sequence $\left(u_{k}\right)_{k}$ is uniformly bounded from below by some positive number:

Proposition 3.1. Let $\left(u_{k}\right)_{k}$ be a sequence of solutions of $\left.E L_{k}\right]$. There exists $\varepsilon_{0}>0$ independent of $k$ such that

$$
u_{k} \geq \varepsilon_{0}
$$

for all $k$.

Proof. We follow the arguments in Hebey-Veronelli [13]. Given $K>0$, we let $H=h+K$ and choose $K$ large enough in order to have $H \geq 1$. For any $\delta>0$ we consider the unique functions $\psi_{k, \delta}, \psi_{\delta}$ and $\psi_{0}$ solving:

$$
\left\{\begin{array}{l}
\triangle_{g} \psi_{k, \delta}+H \psi_{k, \delta}=a_{k}-\delta f^{-}, \\
\triangle_{g} \psi_{\delta}+H \psi_{\delta}=a-\delta f^{-}, \\
\triangle_{g} \psi_{0}+H \psi_{0}=a,
\end{array}\right.
$$

where we have let $f^{-}=-\min (f, 0)$. By standard elliptic theory, $\psi_{k, \delta} \rightarrow \psi_{\delta}$ in $C^{0}(M)$ as $k \rightarrow \infty$ and $\psi_{\delta} \rightarrow \psi_{0}$ in $C^{0}(M)$ as $\delta \rightarrow 0$. Since $a \not \equiv 0$, by the maximum principle there holds $\psi_{0}>0$ in $M$ and so, for some $\delta_{0}$ small enough, $\psi_{\delta_{0}}>0$ in $M$. In particular, $\psi_{k, \delta_{0}} \geq \frac{1}{2} \psi_{\delta_{0}}$ for $k$ large enough. Consider now $\theta_{k}=t \psi_{k, \delta_{0}}, t>0$. Since $q_{k} \rightarrow 2^{*}$ and $a_{k} \rightarrow a$ in $C^{0}(M)$ as $k \rightarrow \infty$, for $t$ sufficiently small (that does not depend on $k$ ) there holds for any $k$ large enough:

$$
\triangle_{g} \theta_{k}+H \theta_{k} \leq \frac{a_{k}}{\theta_{k}^{q_{k}+1}}-f^{-} \theta_{k}^{q_{k}-1} .
$$

Hence:

$$
\begin{aligned}
\triangle_{g}\left(u_{k}-\theta_{k}\right)+H\left(u_{k}-\theta_{k}\right) & \geq f u_{k}^{q_{k}-1}+f^{-} \theta_{k}^{q_{k}-1}+a_{k}\left(u_{k}^{-q_{k}-1}-\theta_{k}^{-q_{k}-1}\right) \\
& \geq 0
\end{aligned}
$$

at any point $x \in M$ such that $u_{k}(x) \leq \theta_{k}(x)$. By the maximum principle, we thus have $u_{k} \geq \theta_{k}$ in $M$. Since $\theta_{k} \geq \frac{1}{2} t \psi_{\delta_{0}}$, we have a uniform lower bound for the $u_{k}$. 
With (3.4) we get in particular the existence of some constant $C$ depending on $h, f, a$ and $\varepsilon_{0}$ such that

$$
\left|\triangle_{g} u_{k}\right| \leq C u_{k}^{q_{k}-1}
$$

Standard elliptic theory and (3.7) show that Theorem 2.1] is proved provided $\left(u_{k}\right)_{k}$ is uniformly bounded in $L^{\infty}(M)$. We thus proceed by contradiction and assume that

$$
\max _{M} u_{k} \rightarrow+\infty
$$

as $k \rightarrow \infty$. In this section we perform an asymptotic analysis of the sequence $\left(u_{k}\right)_{k}$ around a concentration point and obtain sharp pointwise estimates. We denote the injectivity radius of $(M, g)$ by $i_{g}$. Following Druet-Hebey [10, we let $\left(x_{k}\right)_{k}$ be a sequence of points in $M$ and $\left(\rho_{k}\right)_{k}$ be a sequence of positive numbers, with $0<\rho_{k}<i_{g} / 7$, satisfying for any $k \in \mathbb{N}$ :

$$
\left\{\begin{array}{l}
x_{k} \text { is a critical point of } u_{k}, \\
d_{g}\left(x_{k}, x\right)^{\frac{2}{q_{k}-2}} u_{k}(x) \leq C \text { for all } x \in B_{x_{k}}\left(7 \rho_{k}\right), \\
\lim _{k \rightarrow \infty} \rho_{k}^{\frac{2}{q_{k}-2}} \sup _{B_{x_{k}}\left(6 \rho_{k}\right)} u_{k}=+\infty .
\end{array}\right.
$$

In (3.9) and in everything that follows we will denote by $C$ some constant that does not depend on $k$. We define $\mu_{k}$ as

$$
\mu_{k}=u_{k}\left(x_{k}\right)^{-\frac{q_{k}-2}{2}} .
$$

We can apply here the asymptotic analysis of equation $E L_{k}$ and of subcritical perturbations of the Yamabe equation as found respectively in Druet-Hebey [10] and Druet [9]. Similar a priori blow-up techniques were first developed for the analysis of compactness of solutions of the Yamabe equation by Schoen [25, Li-Zhu [16] and by Druet [9]. Assuming (3.9) and (3.3) we obtain that

$$
\mu_{k}^{-\frac{2}{q_{k}-2}} \sim \sup _{B_{x_{k}}\left(6 \rho_{k}\right)} u_{k}, \quad \frac{\rho_{k}}{\mu_{k}} \rightarrow+\infty
$$

(so, in particular, $\mu_{k} \rightarrow 0$ as $k \rightarrow \infty$ ), that

$$
f\left(x_{0}\right)>0,
$$

where $x_{0}$ is a limit of a subsequence of $x_{k}$, and that

$$
\mu_{k}^{\frac{2}{q_{k}-2}} u_{k}\left(\exp _{x_{k}}\left(\mu_{k} x\right)\right) \rightarrow\left(1+\frac{f\left(x_{0}\right)}{n(n-2)}|x|^{2}\right)^{-\frac{n-2}{2}}
$$

in $C_{l o c}^{1}\left(\mathbb{R}^{n}\right)$ as $k \rightarrow \infty$, where $\mu_{k}$ is as in (3.10). Also the Harnack inequality stated in DruetHebey [10] (lemma 1.3) is still satisfied here: there exists $C>1$ such that for any sequence $\left(s_{k}\right)_{k}$ of positive real numbers with $0<6 s_{k} \leq \rho_{k}$ we have

$$
s_{k}\left\|\nabla u_{k}\right\|_{L^{\infty}\left(\Omega_{k}\right)} \leq C \sup _{\Omega_{k}} u_{k} \leq C^{2} \inf _{\Omega_{k}} u_{k},
$$

where $\Omega_{k}=B_{x_{k}}\left(6 s_{k}\right) \backslash B_{x_{k}}\left(\frac{1}{6} s_{k}\right)$. Now we define $\varphi_{k}:\left(0, \rho_{k}\right) \mapsto \mathbb{R}^{+}$as the mean value of $u_{k}$ on spheres centered at $x_{k}$ :

$$
\varphi_{k}(r)=\frac{1}{\left|\partial B_{x_{k}}(r)\right|_{g}} \int_{\partial B_{x_{k}}(r)} u_{k} d \sigma_{g}
$$

Also we define $r_{k}$, the radius of influence of the bubble centered at $x_{k}$ :

$$
r_{k}=\sup \left\{r \in\left(2 R_{0} \mu_{k} ; \rho_{k}\right) \text { such that } s^{\frac{2}{q_{k}-2}} \varphi_{k}(s) \text { is nonincreasing in }\left(2 R_{0} \mu_{k} ; r\right)\right\} \text {, }
$$


where we have let

$$
R_{0}^{2}=\frac{n(n-2)}{f\left(x_{0}\right)}
$$

With (3.12), for any positive $R$ there holds, as $k \rightarrow \infty$ :

$$
\left(R \mu_{k}\right)^{\frac{2}{q_{k}-2}} \varphi_{k}\left(R \mu_{k}\right) \rightarrow R^{\frac{n-2}{2}}\left(1+\frac{R^{2}}{R_{0}^{2}}\right)^{1-\frac{n}{2}},
$$

where $R_{0}$ is as in (3.16). Hence

$$
\frac{r_{k}}{\mu_{k}} \rightarrow+\infty
$$

Note that from the definition of $r_{k}$ there holds that

$$
r^{\frac{2}{q_{k}-2}} \varphi_{k} \text { is nonincreasing in }\left(2 R_{0} \mu_{k}, r_{k}\right)
$$

and that

$$
\text { if } r_{k}<\rho_{k},\left(r^{\frac{2}{q_{k}-2}} \varphi_{k}(r)\right)^{\prime}\left(r_{k}\right)=0 .
$$

As consequence of (3.13) one has that for $0<s \leq r_{k}$ and for some positive constant $C$ :

$$
\frac{1}{C} \sup _{B_{x_{k}}(6 s) \backslash B_{x_{k}}\left(\frac{1}{6} s\right)} u_{k} \leq \varphi_{k}(s) \leq C \inf _{B_{x_{k}}(6 s) \backslash B_{x_{k}}\left(\frac{1}{6} s\right)} u_{k} .
$$

In particular, by (3.19), there holds for any $R>0$ :

$$
\sup _{x \in B_{x_{k}}\left(6 r_{k}\right) \backslash B_{x_{k}}\left(R \mu_{k}\right)} d_{g}\left(x_{k}, x\right)^{\frac{2}{q_{k}-2}} u_{k}(x) \leq\left(R \mu_{k}\right)^{\frac{2}{q_{k}-2}} \varphi_{k}\left(R \mu_{k}\right)
$$

so that, with (3.17):

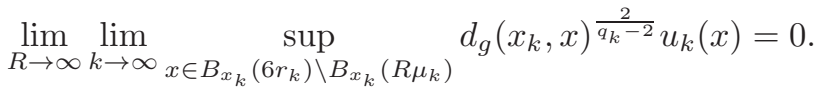

Let us define

$$
\eta_{k}=\sup _{B_{x_{k}}\left(6 r_{k}\right) \backslash B_{x_{k}}\left(\frac{1}{6} r_{k}\right)} u_{k} .
$$

In particular (3.13), (3.22) and (3.4) imply that:

$$
r_{k}^{2} \eta_{k}^{q_{k}-2} \rightarrow 0 \text { and } r_{k} \rightarrow 0 .
$$

Now we prove a sharp asymptotic control from above on $u_{k}$ on a ball of radius $r_{k}$.

Lemma 3.2. Let $\left(u_{k}\right)_{k}$ be a sequence of solutions of EL satisfying (3.3) and (3.8). Let $\left(x_{k}\right)_{k}$ and $\left(\rho_{k}\right)_{k}$ be such that (3.9) holds. There exists a positive constant $C$ such that, for any $x \in B_{x_{k}}\left(6 r_{k}\right) \backslash\left\{x_{k}\right\}$ :

$$
u_{k}(x)+d_{g}\left(x_{k}, x\right)\left|\nabla u_{k}(x)\right| \leq C \mu_{k}^{n-\frac{2\left(q_{k}-1\right)}{q_{k}-2}} d_{g}\left(x_{k}, x\right)^{2-n} .
$$

As a consequence:

$$
r_{k}^{n-2}=O\left(\mu_{k}^{n-\frac{2\left(q_{k}-1\right)}{q_{k}-2}}\right)
$$

where $\mu_{k}$ is as in (3.10. 
Proof. We first show that there exists a positive constant $C$ such that

$$
u_{k}(x) \leq C\left(\mu_{k}^{n-\frac{2\left(q_{k}-1\right)}{q_{k}-2}} d_{g}\left(x_{k}, x\right)^{2-n}+\eta_{k}\right)
$$

for all $x \in B_{x_{k}}\left(6 r_{k}\right) \backslash\left\{x_{k}\right\}$ and for all $k$, where $\eta_{k}$ is as in (3.23). To prove this we let $\left(y_{k}\right)_{k}$ be an arbitrary sequence in $B_{x_{k}}\left(6 r_{k}\right) \backslash\left\{x_{k}\right\}$ and prove that there exists a positive constant $C$ such that, up to a subsequence:

$$
u_{k}\left(y_{k}\right)=O\left(\mu_{k}^{n-\frac{2\left(q_{k}-1\right)}{q_{k}-2}} d_{g}\left(x_{k}, y_{k}\right)^{2-n}\right)+O\left(\eta_{k}\right) .
$$

First, (3.28) follows from (3.12) if $d_{g}\left(x_{k}, y_{k}\right)=O\left(\mu_{k}\right)$ and from (3.13) if $\frac{d_{g}\left(x_{k}, y_{k}\right)}{r_{k}} \not \rightarrow 0$ when $k \rightarrow \infty$. We thus assume from now on that

$$
\frac{d_{g}\left(x_{k}, y_{k}\right)}{\mu_{k}} \rightarrow+\infty \text { and } \frac{d_{g}\left(x_{k}, y_{k}\right)}{r_{k}} \rightarrow 0 .
$$

We let $G_{k}$ be the Green function of $\triangle_{g}$ in the ball $B_{x_{k}}\left(6 r_{k}\right)$ with Dirichlet boundary condition satisfying $G \geq 1$. We recall that there exists a continuous function $\tau: \mathbb{R}^{+} \rightarrow \mathbb{R}^{+}, \tau(0)=0$ such that

$$
\left|G_{k}(x, y)-\frac{1}{(n-2) \omega_{n-1} d_{g}(x, y)^{n-2}}\right| \leq \tau\left(d_{g}(x, y)\right) d_{g}(x, y)^{2-n}
$$

and

$$
|| \nabla G_{k}(x, y)\left|-\frac{1}{\omega_{n-1} d_{g}(x, y)^{n-1}}\right| \leq \tau\left(d_{g}(x, y)\right) d_{g}(x, y)^{1-n}
$$

(see Aubin 22 or Robert 23] for such estimates). The representation formula for the Green function gives, with (3.7), (3.29) and (3.30):

$$
\begin{aligned}
u_{k}\left(y_{k}\right) & =\int_{B_{x_{k}}\left(6 r_{k}\right)} G_{k}\left(x, y_{k}\right) \triangle_{g} u_{k}(x) d v_{g}(x)-\int_{\partial B_{x_{k}}\left(6 r_{k}\right)} \partial_{\nu} G_{k}\left(x, y_{k}\right) u_{k}(x) d \sigma_{g}(x) \\
& =O\left(\int_{B_{x_{k}}\left(6 r_{k}\right)} d_{g}\left(x, y_{k}\right)^{2-n} u_{k}^{q_{k}-1}(x) d v_{g}(x)\right)+O\left(\eta_{k}\right) .
\end{aligned}
$$

Combining the subcritical analysis in Druet [9] and the analysis for the critical equation in Druet-Hebey [10] one gets that for any $\varepsilon>0$ there exists a positive constant $C_{\varepsilon}$ such that

$$
u_{k}(x) \leq C_{\varepsilon}\left(\mu_{k}^{\frac{2}{q_{k}-2}(1-2 \varepsilon)} d_{g}\left(x_{k}, x\right)^{-(1-\varepsilon) \frac{4}{q_{k}-2}}+\eta_{k}\left(\frac{r_{k}}{d_{g}\left(x_{k}, x\right)}\right)^{\frac{4}{q_{k}-2} \varepsilon}\right)
$$

for all $x \in B_{x_{k}}\left(6 r_{k}\right) \backslash\left\{x_{k}\right\}$. Coming back to (3.32), we have

$$
\begin{aligned}
\int_{B_{x_{k}}\left(6 r_{k}\right)} d_{g}\left(x, y_{k}\right)^{2-n} u_{k}^{q_{k}-1}(x) d v_{g}(x) & =\int_{B_{x_{k}}\left(\mu_{k}\right)} d_{g}\left(x, y_{k}\right)^{2-n} u_{k}^{q_{k}-1}(x) d v_{g}(x) \\
& +\int_{B_{x_{k}}\left(6 r_{k}\right) \backslash B_{x_{k}}\left(\mu_{k}\right)} d_{g}\left(x, y_{k}\right)^{2-n} u_{k}^{q_{k}-1}(x) d v_{g}(x) .
\end{aligned}
$$

One finds, on one hand using (3.12) and 3.29) that

$$
\int_{B_{x_{k}}\left(\mu_{k}\right)} d_{g}\left(y_{k}, x\right)^{2-n} u_{k}^{q_{k}-1}(x) d v_{g}(x)=O\left(\mu_{k}^{n-\frac{2\left(q_{k}-1\right)}{q_{k}-2}} d_{g}\left(x_{k}, y_{k}\right)^{2-n}\right)
$$


and on the other hand using (3.33) and (3.29) that

$$
\begin{aligned}
& \int_{B_{x_{k}}\left(6 r_{k}\right) \backslash B_{x_{k}}\left(\mu_{k}\right)} d_{g}\left(y_{k}, x\right)^{2-n} u_{k}^{q_{k}-1}(x) d v_{g}(x) \\
& =O\left(\mu_{k}^{\frac{2\left(q_{k}-1\right)}{q_{k}-2}(1-2 \varepsilon)} \int_{B_{x_{k}}\left(6 r_{k}\right) \backslash B_{x_{k}}\left(\mu_{k}\right)} d_{g}\left(y_{k}, x\right)^{2-n} d_{g}\left(x_{k}, x\right)^{-\frac{4\left(q_{k}-1\right)}{q_{k}-2}(1-\varepsilon)} d v_{g}(x)\right) \\
& \quad+O\left(\int_{B_{x_{k}}\left(6 r_{k}\right) \backslash B_{x_{k}}\left(\mu_{k}\right)} \eta_{k}^{q_{k}-1} r_{k}^{\frac{4\left(q_{k}-1\right)}{q_{k}-2} \varepsilon} d_{g}\left(y_{k}, x\right)^{2-n} d_{g}\left(x_{k}, x\right)^{-\frac{4\left(q_{k}-1\right)}{q_{k}-2} \varepsilon} d v_{g}(x)\right) \\
& =O\left(\mu_{k}^{n-\frac{2\left(q_{k}-1\right)}{q_{k}-2}} d_{g}\left(x_{k}, y_{k}\right)^{2-n}\right)+O\left(r_{k}^{2} \eta_{k}^{q_{k}-1}\right) \\
& =O\left(\mu_{k}^{n-\frac{2\left(q_{k}-1\right)}{q_{k}-2}} d_{g}\left(x_{k}, y_{k}\right)^{2-n}\right)+o\left(\eta_{k}\right),
\end{aligned}
$$

where we used (3.24) to obtain the last equality in (3.36). In the end, (3.32), (3.34), (3.35) and (3.36) yield (3.28) and in particular (3.27) holds true. Now we show that there exists a positive constant $C$ such that for $k$ large enough:

$$
\eta_{k} \leq C \mu_{k}^{n-\frac{2\left(q_{k}-1\right)}{q_{k}-2}} r_{k}^{2-n} .
$$

Using (3.19) we can assert that for any $0<\delta<1$ :

$$
\left(\delta r_{k}\right)^{\frac{2}{q_{k}-2}} \varphi_{k}\left(\delta r_{k}\right) \geq r_{k}^{\frac{2}{q_{k}-2}} \varphi_{k}\left(r_{k}\right) .
$$

So (3.21), (3.38) and the definition of $\varphi_{k}$ as in (3.14) give, for some positive constant $C$ :

$$
\frac{1}{C} r_{k}^{\frac{2}{q_{k}-2}} \eta_{k} \leq r_{k}^{\frac{2}{q_{k}-2}} \varphi_{k}\left(r_{k}\right) \leq\left(\delta r_{k}\right)^{\frac{2}{q_{k}-2}} \sup _{\partial B_{x_{k}}\left(\delta r_{k}\right)} u_{k}
$$

where $\eta_{k}$ is as in (3.23). Using (3.27) one gets for some positive $C$ :

$$
\frac{1}{C} \eta_{k} \leq \delta^{\frac{2}{q_{k}-2}}\left(\mu_{k}^{n-\frac{2\left(q_{k}-1\right)}{q_{k}-2}}\left(\delta r_{k}\right)^{2-n}+\eta_{k}\right)
$$

We just need to choose $\delta$ small enough to have $C \delta^{\frac{2}{q_{k}-2}}<1$ to obtain

$$
\eta_{k} \leq C \mu_{k}^{n-\frac{2\left(q_{k}-1\right)}{q_{k}-2}}\left(\delta r_{k}\right)^{2-n}
$$

for some positive $C$, which shows (3.37). Then (3.37) and (3.27) conclude the sharp asymptotic (3.25) for $u_{k}$. The gradient part in (3.25) is obtained choosing $s_{k}=d_{g}\left(x_{k}, y_{k}\right)$, for any sequence $y_{k} \in B_{x_{k}}\left(6 r_{k}\right)$, in (3.13). Finally (3.26) is obtained using (3.4) in (3.25), and this concludes the proof of Lemma 3.2

With Lemma 3.2 we now determine the asymptotical behavior of the sequence $\left(u_{k}\right)_{k}$ at the boundary of its maximal ball of influence:

Lemma 3.3. Let $\left(u_{k}\right)_{k}$ be a sequence of solutions of (ELk) satisfying (3.3) and (3.8). Let $\left(x_{k}\right)_{k}$ and $\left(\rho_{k}\right)_{k}$ be such that (3.9) holds true. Then up to a subsequence, we have $\rho_{k}=r_{k}, \rho_{k} \rightarrow 0$, $\rho_{k}^{\frac{2}{q_{k}-2}} u_{k}\left(x_{k}\right) \rightarrow+\infty$ and there exists a harmonic function $H$ in $B_{0}(5)$ with $H(0) \leq 0$ such that

$$
\mu_{k}^{\frac{2\left(q_{k}-1\right)}{q_{k}-2}-n} \rho_{k}^{n-2} u_{k}\left(\exp _{x_{k}}\left(\rho_{k} x\right)\right) \rightarrow \frac{R_{0}^{n-2}}{|x|^{n-2}}+H(x)
$$

in $C_{l o c}^{2}\left(B_{0}(5) \backslash\{0\}\right)$ as $k \rightarrow \infty$, where $R_{0}$ is as in (3.16). 
Remark 3.4. With the definition of $\mu_{k}$ in (3.10) one can also write (3.39) as

$$
u_{k}\left(x_{k}\right)^{1-n+q_{k} \frac{n-2}{2}} \rho_{k}^{n-2} u_{k}\left(\exp _{x_{k}}\left(\rho_{k} x\right)\right) \rightarrow \frac{R_{0}^{n-2}}{|x|^{n-2}}+H(x) .
$$

Proof. For $x \in B_{0}(5)$ we define

$$
\begin{aligned}
& \hat{u}_{k}(x)=\mu_{k}^{\frac{2\left(q_{k}-1\right)}{q_{k}-2}-n} r_{k}^{n-2} u_{k}\left(\exp _{x_{k}}\left(r_{k} x\right)\right), \\
& \hat{g}_{k}(x)=\left(\exp _{x_{k}}^{*}\right) g\left(r_{k} x\right) .
\end{aligned}
$$

Since $r_{k} \rightarrow 0$ by (3.24), $\hat{g}_{k} \rightarrow \xi$ the euclidean metric in $C_{l o c}^{2}\left(B_{0}(5)\right)$. Thanks to (3.25) we also have that

$$
\hat{u}_{k}(x) \leq C|x|^{2-n}
$$

in $B_{0}(5) \backslash\{0\}$. By $E L_{k}$ and (3.41) we therefore obtain that for all $k$,

$$
\triangle_{\hat{g}_{k}} \hat{u}_{k}=\hat{F}_{k}(x)
$$

in $B_{0}(5) \backslash\{0\}$, where

$$
\left|\hat{F}_{k}(x)\right| \leq C\left(\frac{\mu_{k}}{r_{k}}\right)^{(n-2)\left(q_{k}-2\right)-2}|x|^{\left(q_{k}-1\right)(2-n)}
$$

for some positive $C$. By standard elliptic theory, (3.42), (3.43) and by (3.18) there exists $\hat{U}$ harmonic in $B_{0}(5) \backslash\{0\}$ such that $\hat{u}_{k} \rightarrow \hat{U}$ in $C_{l o c}^{2}\left(B_{0}(5) \backslash\{0\}\right)$. Since by (3.41) $\hat{U}$ satisfies that $0 \leq \hat{U}(x) \leq C|x|^{2-n}$ for some positive constant $C$ in $B_{0}(5) \backslash\{0\}$ we can write

$$
\hat{U}(x)=\frac{\lambda}{|x|^{n-2}}+H(x),
$$

where $\lambda \geq 0$ and $H$ is harmonic in $B_{0}(5)$. To show that $\lambda=R_{0}^{n-2}$ we integrate (3.42) on $B_{0}(1)$ : there holds

$$
-\int_{\partial B_{0}(1)} \partial_{\nu} \hat{u}_{k} d \sigma_{\hat{g}_{k}}=\int_{B_{0}(1)} \hat{F}_{k} d v_{\hat{g}_{k}} .
$$

Straightforward calculation gives, using (3.12) and (3.25):

$$
\begin{aligned}
\int_{B_{0}(1)} \hat{F}_{k} d v_{\hat{g}_{k}}= & \int_{B_{0}(1)}\left(\frac{\mu_{k}}{r_{k}}\right)^{(n-2)\left(q_{k}-2\right)-2} f\left(\exp _{x_{k}}\left(r_{k} x\right)\right) \hat{u}_{k}^{q_{k}-1}(x) d x+o(1) \\
& =f\left(x_{0}\right) \int_{\mathbb{R}^{n}}\left(1+\frac{|x|^{2}}{R_{0}^{2}}\right)^{-1-\frac{n}{2}} d x+o(1) . \\
& =(n-2) \omega_{n-1} R_{0}^{n-2}+o(1) .
\end{aligned}
$$

On the other side (3.44) shows that

$$
\int_{\partial B_{0}(1)} \partial_{\nu} \hat{u}_{k} d \sigma_{\hat{g}_{k}} \rightarrow-\lambda(n-2) \omega_{n-1}
$$

as $k \rightarrow \infty$. So (3.46) and (3.47) in 3.45) yield $\lambda=R_{0}^{n-2}$. In the end,

$$
\mu_{k}^{\frac{2\left(q_{k}-1\right)}{q_{k}-2}-n} r_{k}^{n-2} u_{k}\left(\exp _{x_{k}}\left(r_{k} x\right)\right) \rightarrow \hat{U}=\frac{R_{0}^{n-2}}{|x|^{n-2}}+H(x)
$$

in $C_{l o c}^{2}\left(B_{0}(5) \backslash\{0\}\right)$ as $k \rightarrow \infty$. We now prove that $r_{k}=\rho_{k}$. Assume first by contradiction that $r_{k}<\rho_{k}$. Let for any $r>0$ :

$$
\varphi(r)=\frac{1}{\omega_{n-1} r^{n-1}} \int_{\partial B_{0}(r)} \hat{U} d \sigma
$$


where $\hat{U}$ is as in (3.44). On one side, 3.48 shows that

$$
\varphi(r)=\left(\frac{R_{0}}{r}\right)^{n-2}+H(0) .
$$

On the other side (3.20) and (3.48) give us that

$$
\left(r^{\frac{n-2}{2}} \varphi(r)\right)^{\prime}(1)=0
$$

Combining (3.49) and (3.50) yields

$$
H(0)=R_{0}^{n-2} \text {. }
$$

To conclude the proof of Lemma 3.3 and show that $r_{k}=\rho_{k}$ we therefore show that $H(0) \leq 0$. We let $X_{k}(x)=\nabla f_{k}(x)$, where $f_{k}(x)=\frac{1}{2} d_{g}\left(x_{k}, x\right)^{2}$, be the vector field whose coordinates in the exponential chart at $x_{k}$ are $x^{i}$ and use the Pohozaev identity as in Druet-Hebey [10, (see also Druet [9]) on $\Omega_{k}=B_{x_{k}}\left(6 r_{k}\right)$ :

$$
\begin{aligned}
& \int_{\Omega_{k}} \nabla u_{k}\left(X_{k}\right) \triangle_{g} u_{k} d v_{g}=\int_{\Omega_{k}}\left(\nabla^{\sharp} X_{k}\left(\nabla u_{k}, \nabla u_{k}\right)-\frac{1}{2}\left(\operatorname{div}_{g} X_{k}\right)\left|\nabla u_{k}\right|^{2}\right) d v_{g} \\
& +\int_{\partial \Omega_{k}}\left(\frac{1}{2}\left(X_{k}, \nu\right)_{g}\left|\nabla u_{k}\right|^{2}-\nabla u_{k}\left(X_{k}\right) \partial_{\nu} u_{k}\right) d \sigma_{g},
\end{aligned}
$$

where we have let $\left(\nabla^{\sharp} X_{k}\right)^{i j}=\left(\nabla^{i} X_{k}\right)^{j}$, where $(\cdot, \cdot)_{g}$ is the scalar product defined by $g$ and $\nu$ is the unit outward normal to $\partial \Omega_{k}$. In the exponential chart at $x_{k}$ we have

$$
\left(\nabla^{i} X_{k}\right)^{j}=g^{i j}+O\left(d_{g}\left(x_{k}, x\right)^{2}\right)
$$

and so integrating by parts the first integral in the right-hand side of (3.51) and using (3.52) gives

$$
\begin{aligned}
& \int_{\Omega_{k}}\left(\nabla u_{k}\left(X_{k}\right)+\frac{n-2}{2} u_{k}\right) \triangle_{g} u_{k} d v_{g}=O\left(\int_{\Omega_{k}} d_{g}\left(x_{k}, x\right)^{2}\left|\nabla u_{k}\right|^{2} d v_{g}\right) \\
& +\int_{\partial \Omega_{k}}\left(\frac{1}{2}\left(X_{k}, \nu\right)_{g}\left|\nabla u_{k}\right|^{2}-\nabla u_{k}\left(X_{k}\right) \partial_{\nu} u_{k}-\frac{n-2}{2} u_{k} \partial_{\nu} u_{k}\right) d \sigma_{g} .
\end{aligned}
$$

Using (3.48) it is easily seen that, since $H$ is harmonic:

$$
\begin{aligned}
& \mu_{k}^{\frac{4\left(q_{k}-1\right)}{q_{k}-2}-2 n} r_{k}^{n-2} \int_{\partial \Omega_{k}}\left(\frac{1}{2}\left(X_{k}, \nu\right)_{g}\left|\nabla u_{k}\right|^{2}-\nabla u_{k}\left(X_{k}\right) \partial_{\nu} u_{k}-\frac{n-2}{2} u_{k} \partial_{\nu} u_{k}\right) d \sigma_{g} \\
& =\int_{\partial B_{0}(1)}\left(\frac{1}{2}|\nabla \hat{U}|^{2}-\left|\partial_{\nu} \hat{U}\right|^{2}-\frac{n-2}{2} \hat{U} \partial_{\nu} \hat{U}\right) d x+o(1) \\
& =\frac{(n-2)^{2}}{2} R_{0}^{n-2} \omega_{n-1} H(0)+\int_{\partial B_{0}(1)}\left(\frac{1}{2}|\nabla H|^{2}-\left|\partial_{\nu} H\right|^{2}-\frac{n-2}{2} H \partial_{\nu} H\right) d x+o(1) .
\end{aligned}
$$

A Pohozaev identity like (3.53) for $H$ and $X=x$ applied to the euclidean ball $B_{0}(1)$ for the euclidean metric gives, since $H$ is harmonic:

$$
\begin{aligned}
\int_{\partial B_{0}(1)} & \left(\frac{1}{2}|\nabla H|^{2}-\left|\partial_{\nu} H\right|^{2}-\frac{n-2}{2} H \partial_{\nu} H\right) d x \\
= & \int_{B_{0}(1)}\left(\nabla H(X)+\frac{n-2}{2} H\right) \triangle_{\xi} H d x=0 .
\end{aligned}
$$


We finally obtain, with (3.54) and (3.55):

$$
\begin{aligned}
& \int_{\partial \Omega_{k}}\left(\frac{1}{2}\left(X_{k}, \nu\right)_{g}\left|\nabla u_{k}\right|^{2}-\nabla u_{k}\left(X_{k}\right) \partial_{\nu} u_{k}-\frac{n-2}{2} u_{k} \partial_{\nu} u_{k}\right) d \sigma_{g} \\
& =\left(\frac{(n-2)^{2}}{2} R_{0}^{n-2} \omega_{n-1} H(0)+o(1)\right) \mu_{k}^{2 n-\frac{4\left(q_{k}-1\right)}{q_{k}-2}} r_{k}^{2-n}
\end{aligned}
$$

We write now:

$$
\int_{\Omega_{k}} d_{g}\left(x_{k}, x\right)^{2}\left|\nabla u_{k}\right|^{2} d v_{g}=\int_{B_{x_{k}}\left(\mu_{k}\right)} d_{g}\left(x_{k}, x\right)^{2}\left|\nabla u_{k}\right|^{2} d v_{g}+\int_{B_{x_{k}}\left(r_{k}\right) \backslash B_{x_{k}}\left(\mu_{k}\right)} d_{g}\left(x_{k}, x\right)^{2}\left|\nabla u_{k}\right|^{2} d v_{g}
$$

so that, using (3.12) and (3.25) we obtain:

$$
\int_{\Omega_{k}} d_{g}\left(x_{k}, x\right)^{2}\left|\nabla u_{k}\right|^{2} d v_{g}= \begin{cases}O\left(\mu_{k}{ }^{-\frac{4\left(q_{k}-1\right)}{q_{k}-2}} r_{k}\right) & \text { if } n=3, \\ O\left(\mu_{k}^{8-\frac{4\left(q_{k}-1\right)}{q_{k}-2}} \ln \frac{r_{k}}{\mu_{k}}\right) & \text { if } n=4, \\ O\left(\mu_{k}^{4+n-\frac{4\left(q_{k}-1\right)}{q_{k}-2}}\right) & \text { if } n \geq 5 .\end{cases}
$$

With (3.26) equation (3.57) becomes:

$$
\int_{\Omega_{k}} d_{g}\left(x_{k}, x\right)^{2}\left|\nabla u_{k}\right|^{2} d v_{g}=o\left(\mu_{k}^{2 n-\frac{4\left(q_{k}-1\right)}{q_{k}-2}} r_{k}^{2-n}\right)
$$

for $3 \leq n \leq 5$. Now we compute the left-hand side integral in (3.53). Using $\left.E L_{k}\right)$ and integrating by parts yield:

$$
\begin{aligned}
& \int_{\Omega_{k}}\left(\nabla u_{k}\left(X_{k}\right)+\frac{n-2}{2} u_{k}\right) \triangle_{g} u_{k} d v_{g}=n\left(\frac{1}{2^{*}}-\frac{1}{q_{k}}\right) \int_{\Omega_{k}} f u_{k}^{q_{k}} d v_{g} \\
& -\frac{1}{q_{k}} \int_{\Omega_{k}} \nabla f\left(X_{k}\right) u_{k}^{q_{k}} d v_{g}+O\left(r_{k} \int_{\partial \Omega_{k}} u_{k}^{q_{k}} d \sigma_{g}\right)+O\left(\int_{\Omega_{k}} d_{g}\left(x_{k}, x\right)^{2} u_{k}^{q_{k}} d v_{g}\right) \\
& +O\left(\int_{\Omega_{k}}\left(d_{g}\left(x_{k}, x\right)\left|\nabla u_{k}\right|+u_{k}\right)\left(u_{k}^{-q_{k}-1}+u_{k}\right) d v_{g}\right) .
\end{aligned}
$$

Using (3.4), (3.12) and (3.25), the same computation as for (3.57) gives, since $3 \leq n \leq 5$ :

$$
\int_{\Omega_{k}}\left(d_{g}\left(x_{k}, x\right)\left|\nabla u_{k}\right|+u_{k}\right)\left(u_{k}^{-q_{k}-1}+u_{k}+1\right) d v_{g}=o\left(\mu_{k}^{2 n-\frac{4\left(q_{k}-1\right)}{q_{k}-2}} r_{k}^{2-n}\right) .
$$

Using (3.48) we obtain:

$$
r_{k} \int_{\partial \Omega_{k}} u_{k}^{q_{k}} d \sigma_{g}=O\left(\left(\frac{\mu_{k}}{r_{k}}\right)^{(n-2) q_{k}} \mu_{k}^{-\frac{2 q_{k}}{q_{k}-2}} r_{k}^{n}\right)=o\left(\mu_{k}^{2 n-\frac{4\left(q_{k}-1\right)}{q_{k}-2}} r_{k}^{2-n}\right) .
$$

Finally, using once again (3.12), (3.25) and (3.26) there holds:

$$
\int_{\Omega_{k}} d_{g}\left(x_{k}, x\right)^{2} u_{k}^{q_{k}} d v_{g}=O\left(\mu_{k}^{n-\frac{4}{q_{k}-2}}\right)=o\left(\mu_{k}^{2 n-\frac{4\left(q_{k}-1\right)}{q_{k}-2}} r_{k}^{2-n}\right),
$$


where the last equality is once again true because $3 \leq n \leq 5$. By (3.53) and (3.59) and with (3.56), (3.58), (3.60), (3.61) and (3.62) we obtain:

$$
\begin{aligned}
& \frac{1}{q_{k}} \int_{\Omega_{k}} \nabla f\left(X_{k}\right) u_{k}^{q_{k}} d v_{g}+n\left(\frac{1}{q_{k}}-\frac{1}{2^{*}}\right) \int_{\Omega_{k}} f u_{k}^{q_{k}} d v_{g} \\
& \quad=\left(-\frac{(n-2)^{2}}{2} \omega_{n-1} R_{0}^{n-2} H(0)+o(1)\right) \mu_{k}^{2 n-\frac{4\left(q_{k}-1\right)}{q_{k}-2}} r_{k}^{2-n} .
\end{aligned}
$$

By (3.11) we have $f(x)>0$ for $x \in \Omega_{k}$ for $k$ large enough so, since $q_{k} \leq 2^{*}$ and $u_{k} \geq 0$, with (3.63) it is enough to show that

$$
\frac{1}{q_{k}} \int_{\Omega_{k}} \nabla f\left(X_{k}\right) u_{k}^{q_{k}} d v_{g}=o\left(\mu_{k}^{2 n-\frac{4\left(q_{k}-1\right)}{q_{k}-2}} r_{k}^{2-n}\right)
$$

to conclude that $H(0) \leq 0$. We first assume that $n=3$. For $R>1$ we write

$$
\int_{\Omega_{k}} \nabla f\left(X_{k}\right) u_{k}^{q_{k}} d v_{g}=\int_{B_{x_{k}}\left(R \mu_{k}\right)} \nabla f\left(X_{k}\right) u_{k}^{q_{k}} d v_{g}+\int_{B_{x_{k}}\left(r_{k}\right) \backslash B_{x_{k}}\left(R \mu_{k}\right)} \nabla f\left(X_{k}\right) u_{k}^{q_{k}} d v_{g} .
$$

On one side, using (3.12) and since $x \mapsto x^{i}$ is an odd function in $\mathbb{R}^{n}$ for any $i$, we have:

$$
\int_{B_{0}\left(R \mu_{k}\right)} x^{i} \nabla_{i} f(x) u_{k}^{q_{k}}(x) d v_{g}(x)=o\left(\mu_{k}^{n-1-\frac{4}{q_{k}-2}}\right) .
$$

On the other side, using (3.25) yields:

$$
\begin{aligned}
\int_{B_{x_{k}}\left(r_{k}\right) \backslash B_{x_{k}}\left(R \mu_{k}\right)} \nabla f\left(X_{k}\right) u_{k}^{q_{k}} d v_{g} & \leq C \mu_{k}^{n-1-\frac{4}{q_{k}-2}} \int_{B_{0}\left(\frac{r_{k}}{\mu_{k}}\right) \backslash B_{0}(R)}|x|^{1+(2-n) q_{k}} d x \\
& \leq C \mu_{k}^{n-1-\frac{4}{q_{k}-2}} R^{1+n+(2-n) q_{k}}
\end{aligned}
$$

for some positive constant $C$ that depends neither on $R$ nor on $k$. Since $q_{k} \rightarrow 2^{*}$ as $k \rightarrow \infty$ (3.66) and (3.67) show that:

$$
\int_{\Omega_{k}} \nabla f\left(X_{k}\right) u_{k}^{q_{k}} d v_{g}=o\left(\mu_{k}^{n-1-\frac{4}{q_{k}-2}}\right)=o\left(\mu_{k}^{6-\frac{4\left(q_{k}-1\right)}{q_{k}-2}} r_{k}^{-1}\right),
$$

the last equality being true only when $n=3$. So there holds $H(0) \leq 0$ if $n=3$. Now we prove (3.64) when $n=4,5$. Using (3.62) and (3.66) we have:

$$
\begin{aligned}
\int_{\Omega_{k}} \nabla f\left(X_{k}\right) u_{k}^{q_{k}} d v_{g} & =\partial_{i} f\left(x_{k}\right) \int_{\Omega_{k}} x^{i} u_{k}^{q_{k}} d v_{g}+O\left(\int_{\Omega_{k}} d_{g}\left(x_{k}, x\right)^{2} u_{k}^{q_{k}} d v_{g}\right) \\
& =o\left(\mu_{k}^{n-1-\frac{4}{q_{k}-2}}\left|\nabla f\left(x_{k}\right)\right|\right)+o\left(\mu_{k}^{2 n-\frac{4\left(q_{k}-1\right)}{q_{k}-2}} r_{k}^{2-n}\right) .
\end{aligned}
$$

We now show that

$$
\mu_{k}^{n-1-\frac{4}{q_{k}-2}}\left|\nabla f\left(x_{k}\right)\right|=o\left(\mu_{k}^{2 n-\frac{4\left(q_{k}-1\right)}{q_{k}-2}} r_{k}^{2-n}\right)
$$

when $4 \leq n \leq 5$. With (3.69) and (3.63) this will show that $H(0) \leq 0$ when $4 \leq n \leq 5$. To do this we apply another Pohozaev identity to $u_{k}$ in the open set $\Omega_{k}$. Let $Y \in \mathbb{R}^{n}$ be a given vector and let $Y_{k}$ be the local vector field whose coordinates in the exponential chart are $Y_{k}^{i}=Y^{i}$. 
Equality (3.51) becomes:

$$
\begin{aligned}
& \int_{\Omega_{k}} \nabla u_{k}\left(Y_{k}\right) \triangle_{g} u_{k} d v_{g}=\int_{\Omega_{k}}\left(\nabla^{\sharp} Y_{k}\left(\nabla u_{k}, \nabla u_{k}\right)-\frac{1}{2} \operatorname{div}_{g} Y_{k}\left|\nabla u_{k}\right|^{2}\right) d v_{g} \\
& \quad+\int_{\partial \Omega_{k}}\left(\frac{1}{2}\left(Y_{k}, \nu\right)_{g}\left|\nabla u_{k}\right|^{2}-\nabla u_{k}\left(Y_{k}\right) \partial_{\nu} u_{k}\right) d \sigma_{g} .
\end{aligned}
$$

Since $\left(\nabla^{i} Y_{k}\right)^{j}=O\left(d_{g}\left(x_{k}, x\right)\right)$, (3.71) becomes with $\left.E L_{k}\right)$ :

$$
\begin{aligned}
& \int_{\Omega_{k}} \nabla u_{k}\left(Y_{k}\right) f u_{k}^{q_{k}-1} d v_{g}=O\left(\int_{\Omega_{k}} d_{g}\left(x_{k}, x\right)\left|\nabla u_{k}\right|^{2} d v_{g}\right) \\
& \quad+O\left(\int_{\partial \Omega_{k}}\left|\nabla u_{k}\right|^{2} d \sigma_{g}\right)+O\left(\int_{\Omega_{k}}\left|\nabla u_{k}\right|\left(u_{k}^{-q_{k}-1}+u_{k}\right) d v_{g}\right) .
\end{aligned}
$$

Using (3.48) we have

$$
\int_{\partial \Omega_{k}}\left|\nabla u_{k}\right|^{2} d \sigma_{g}=O\left(\mu_{k}^{2 n-\frac{4\left(q_{k}-1\right)}{q_{k}-2}} r_{k}^{1-n}\right)=o\left(\mu_{k}^{2 n-1-\frac{4\left(q_{k}-1\right)}{q_{k}-2}} r_{k}^{2-n}\right) .
$$

Using (3.12), (3.25) and (3.26) we find:

$$
\int_{\Omega_{k}} d_{g}\left(x_{k}, x\right)\left|\nabla u_{k}\right|^{2} d v_{g}=O\left(\mu_{k}^{n-1-\frac{4}{q_{k}-2}}\right)=o\left(\mu_{k}^{2 n-1-\frac{4\left(q_{k}-1\right)}{q_{k}-2}} r_{k}^{2-n}\right),
$$

the last equality being true since $n \leq 5$. Once again, (3.4), (3.12), 3.25) and (3.26) yield

$$
\int_{\Omega_{k}}\left|\nabla u_{k}\right|\left(u_{k}^{-q_{k}-1}+u_{k}+1\right) d v_{g}=O\left(\mu_{k}^{n-1-\frac{4}{q_{k}-2}}\right)=o\left(\mu_{k}^{2 n-1-\frac{4\left(q_{k}-1\right)}{q_{k}-2}} r_{k}^{2-n}\right)
$$

since $4 \leq n \leq 5$. These computations with (3.72) give:

$$
\int_{\Omega_{k}} \nabla u_{k}\left(Y_{k}\right) f u_{k}^{q_{k}-1} d v_{g}=o\left(\mu_{k}^{2 n-1-\frac{4\left(q_{k}-1\right)}{q_{k}-2}} r_{k}^{2-n}\right) .
$$

We now compute the latter integral differently:

$$
\begin{aligned}
\int_{\Omega_{k}} \nabla u_{k}\left(Y_{k}\right) f u_{k}^{q_{k}-1} d v_{g}= & \frac{1}{q_{k}} \int_{\Omega_{k}} f \nabla\left(u_{k}^{q_{k}}\right)\left(Y_{k}\right) d v_{g} \\
= & O\left(\int_{\partial \Omega_{k}} u_{k}^{q_{k}} d \sigma_{g}\right)-\frac{1}{q_{k}} \int_{\Omega_{k}}\left(\operatorname{div}_{g} Y_{k}\right) f u_{k}^{q_{k}} d v_{g} \\
& -\frac{1}{q_{k}} \int_{\Omega_{k}} \nabla f\left(Y_{k}\right) u_{k}^{q_{k}} d v_{g} \\
= & O\left(\int_{\partial \Omega_{k}} u_{k}^{q_{k}} d \sigma_{g}\right)+O\left(\int_{\Omega_{k}} d_{g}\left(x_{k}, x\right) u_{k}^{q_{k}} d v_{g}\right) \\
& -\frac{1}{q_{k}}\left(\nabla f\left(Y_{k}\right)\right)\left(x_{k}\right) \int_{\Omega_{k}} u_{k}^{q_{k}} d v_{g} .
\end{aligned}
$$

Using once again (3.12) and (3.25) yields

$$
\int_{\Omega_{k}} d_{g}\left(x_{k}, x\right) u_{k}^{q_{k}} d v_{g}=O\left(\mu_{k}^{n-1-\frac{4}{q_{k}-2}}\right)=o\left(\mu_{k}^{2 n-1-\frac{4\left(q_{k}-1\right)}{q_{k}-2}} r_{k}^{2-n}\right)
$$

since $4 \leq n \leq 5$ and (3.61) gives

$$
\int_{\partial \Omega_{k}} u_{k}^{q_{k}} d v_{g}=o\left(\mu_{k}^{2 n-\frac{4\left(q_{k}-1\right)}{q_{k}-2}} r_{k}^{1-n}\right)=o\left(\mu_{k}^{2 n-1-\frac{4\left(q_{k}-1\right)}{q_{k}-2}} r_{k}^{2-n}\right) .
$$


Gathering the previous computation in (3.73) gives in the end:

$$
\frac{1}{q_{k}}\left(\nabla f\left(Y_{k}\right)\right)\left(x_{k}\right) \int_{\Omega_{k}} u_{k}^{q_{k}} d v_{g}=o\left(\mu_{k}^{2 n-1-\frac{4\left(q_{k}-1\right)}{q_{k}-2}} r_{k}^{2-n}\right) .
$$

Finally, (3.12) and (3.25) yield, with Lebesgue's dominated convergence theorem:

$$
\mu_{k}^{-n+2+\frac{4}{q_{k}-2}} \int_{\Omega_{k}} u_{k}^{q_{k}} d v_{g}=\int_{\mathbb{R}^{n}}\left(1+\frac{|x|^{2}}{R_{0}^{2}}\right)^{-n} d x+o(1),
$$

so that with (3.74) we obtain:

$$
\mu_{k}^{n-2-\frac{4}{q_{k}-2}}\left(\nabla f\left(Y_{k}\right)\right)\left(x_{k}\right)=o\left(\mu_{k}^{2 n-1-\frac{4\left(q_{k}-1\right)}{q_{k}-2}} r_{k}^{2-n}\right) .
$$

Since $Y_{k}^{i}=Y^{i}$ for all $i$, where $Y$ is an arbitrary vector in $\mathbb{R}^{n}$, (3.75) implies (3.70) and shows that $H(0) \leq 0$ when $4 \leq n \leq 5$. In particular, this shows that $r_{k}=\rho_{k}$ and we have thus proven (3.39). Noticing that then

$$
\rho_{k}^{\frac{2}{q_{k}-2}} u_{k}\left(x_{k}\right)=\left(\frac{r_{k}}{\mu_{k}}\right)^{\frac{2}{q_{k}-2}} \rightarrow+\infty
$$

as $q_{k} \rightarrow 2^{*}$, this ends the proof of Lemma 3.3

Note, as mentioned several times in the proof, that the above asymptotic analysis works only when $n \leq 5$. When $n \geq 6$, counterexamples to Theorem 2.1 for critical perturbations $\left(q_{k}=2^{*}\right.$ for all $k$ ) are known, see Druet-Hebey [10].

\section{Stability under subcritical Perturbations: Proof of Theorem 2.1}

Using the asymptotic description obtained in the previous section we show that concentration points cannot appear and prove Theorem [2.1. Following the analysis in Druet-Hebey [10, see also Druet [9], there exists a positive constant $C$ such that for any $k$ there exists $N_{k} \in \mathbb{N}^{*}$ and $N_{k}$ critical points of $u_{k}$ denoted by $x_{1, k}, \ldots, x_{N_{k}, k}$ such that

$$
d_{g}\left(x_{i, k}, x_{j, k}\right)^{\frac{2}{q_{k}-2}} u_{k}\left(x_{i, k}\right) \geq 1
$$

for all $i, j \in\left\{1, \ldots, N_{k}\right\}, i \neq j$, and

$$
\left(\min _{i=1, \ldots, N_{k}} d_{g}\left(x_{i, k}, x\right)\right)^{\frac{2}{q_{k}-2}} u_{k}(x) \leq C_{1}
$$

for all $x \in M$ and for all $k$. Note that by construction, for any $k$ one finds among the $x_{i, k}$ the maximum point of $u_{k}$. As a consequence, $N_{k} \geq 2$ for $k$ large enough, otherwise thanks to (3.8) the hypothesis (3.9) would be satisfied with $\rho_{k}=\frac{1}{8} i_{g}(M)$ and with $x_{k}$ as the maximum point of $u_{k}$ which is impossible by Lemma 3.3 Also note, with (4.2), that hypothesis (3.9) are satisfied if we choose $x_{k}=x_{i_{k}, k}$ for some $1 \leq i_{k} \leq N_{k}$ and for $\rho_{k}$ such that

$$
7 \rho_{k} \leq \min \left(\min _{1 \leq i \leq N_{k}, i \neq i_{k}} d_{g}\left(x_{k}, x_{i, k}\right), \frac{1}{2} i_{g}(M)\right) .
$$

Let

$$
d_{k}=\min _{1 \leq i<j \leq N_{k}} d_{g}\left(x_{i, k}, x_{j, k}\right)
$$

and assume, up to reordering the $x_{i, k}$, that

$$
d_{k}=d_{g}\left(x_{1, k}, x_{2, k}\right) \leq d_{g}\left(x_{1, k}, x_{3, k}\right) \leq \cdots \leq d_{g}\left(x_{1, k}, x_{N_{k}, k}\right) .
$$

The following result shows that the concentration points are not isolated: 
Lemma 4.1. Let $d_{k}$ be as in (4.3). Then $d_{k} \rightarrow 0$ as $k \rightarrow \infty$.

Proof. We proceed by contradiction and assume that there exists $0<d<i_{g}(M)$ such that for all $1 \leq i<j \leq N_{k}$ and for all $k$ :

$$
d_{g}\left(x_{i, k}, x_{j, k}\right) \geq d .
$$

Since $M$ is compact the sequence $\left(N_{k}\right)_{k}$ is bounded and up to a subsequence we can assume that $N_{k}=N \geq 2$ for $k$ large enough. We let $x_{k}$ be a maximum point of $u_{k}$. By (3.8) and (4.2) there exists $i \in\{1, \cdots N\}$ such that $d_{g}\left(x_{i, k}, x_{k}\right) \rightarrow 0$ when $k \rightarrow \infty$. Hence (4.5) shows that (3.9) is satisfied with $x_{k}$ and $\rho_{k}=\frac{1}{16} d$ which is impossible by Lemma 3.3

Among the critical points $\left(x_{i, k}\right)_{1 \leq i \leq N_{k}}$ we isolate those which are at a finite distance from $x_{1, k}$ when rescaling by a $d_{k}$ factor. For all $R>0$, we let $1 \leq N_{R, k} \leq N_{k}$ be such that

$$
\begin{aligned}
& d_{g}\left(x_{1, k}, x_{i, k}\right) \leq R d_{k} \text { for } 1 \leq i \leq N_{R, k} \text { and } \\
& d_{g}\left(x_{1, k}, x_{i, k}\right)>R d_{k} \text { for } N_{R, k}+1 \leq i \leq N_{k} .
\end{aligned}
$$

By (4.4) $N_{R, k}$ is well defined and we have $N_{R, k}=1$ if $R<1$ and $N_{R, k} \geq 2$ otherwise. Let $R>1$.

By (4.3) the balls $B_{x_{i, k}}\left(\frac{d_{k}}{4}\right)$ and $B_{x_{j, k}}\left(\frac{d_{k}}{4}\right)$ are disjoint and contained in $B_{x_{1, k}}\left((R+1) d_{k}\right)$ for all $1 \leq i<j \leq N_{R, k}$. Writing these inclusions in terms of volumes shows that, for any $R>0, N_{R, k}$ is bounded by some positive constant that does not depend on $k$. To investigate the behavior of $u_{k}$ around $x_{1, k}$ let $0<\delta \leq \frac{1}{2} i_{g}(M)$ and define, for all $x \in B_{0}\left(\frac{\delta}{d_{k}}\right)$ :

$$
\begin{aligned}
& \check{u}_{k}(x)=d_{k}^{\frac{2}{q_{k}-2}} u_{k}\left(\exp _{x_{1, k}}\left(d_{k} x\right)\right), \\
& \check{g}_{k}(x)=\left(\exp _{x_{1, k}}^{*} g\right)\left(d_{k} x\right) .
\end{aligned}
$$

By Lemma 4.1, $\check{g}_{k} \rightarrow \xi$ in $C_{l o c}^{2}\left(\mathbb{R}^{n}\right)$. Using $E L_{k}$ we have in $B_{0}\left(\frac{\delta}{d_{k}}\right)$ :

$$
\triangle_{\check{g}_{k}} \check{u}_{k}=\check{F}_{k}
$$

where $\check{F}_{k}$ satisfies, by (3.7):

$$
\left|\check{F}_{k}\right| \leq C_{0} \check{u}_{k}^{q_{k}-1} .
$$

We finally let, for all $1 \leq i \leq N_{k}$ such that $d_{g}\left(x_{1, k}, x_{i, k}\right) \leq \frac{1}{2} i_{g}(M)$ :

$$
\check{x}_{i, k}=\frac{1}{d_{k}} \exp _{x_{1, k}}^{-1}\left(x_{i, k}\right) \text {. }
$$

Let $R>1,0<r<1$ and let

$$
\Omega_{r, R}=B_{0}(R) \backslash \cup_{i=1}^{N_{2 R, k}} B_{\check{x}_{i, k}}(r) .
$$

Then (4.2) shows that $\check{u}_{k}$ is uniformly bounded in $\Omega_{r, R}$ and mimicking the analysis of (3.13) we obtain a Harnack inequality for $\check{u}_{k}$ in $\Omega_{r, R}$ :

$$
\left\|\nabla \check{u}_{k}\right\|_{L^{\infty}\left(\Omega_{r, R}\right)} \leq D_{r, R} \sup _{\Omega_{r, R}} \check{u}_{k} \leq D_{r, R}^{2} \inf _{\Omega_{r, R}} \check{u}_{k},
$$

where $D_{r, R}>1$ is a constant depending only on $r$ and $R$. We have the following result that determines the behavior of $\check{u}_{k}$ around the critical points $\check{x}_{i, k}$ which are $d_{k}$-close to $\check{x}_{1, k}$.

Lemma 4.2. Let $\left(u_{k}\right)_{k}$ be a sequence of solutions of (EL $)$, hence satisfying (3.4), such that (3.3) and (3.8) hold. Let $d_{k}$ be as in (4.3), $\check{x}_{1, k}, \ldots, \check{x}_{N_{k}, k}$ as in (4.10) and $\check{u}_{k}$ as in (4.7). Then only one of the two following situations occurs: 
- either for any $1 \leq i \leq N_{k}$ such that $d_{g}\left(x_{1, k}, x_{i, k}\right)=O\left(d_{k}\right)$ there holds

$$
\sup _{B_{\check{x}_{i, k}}\left(\frac{1}{2}\right)} \check{u}_{k}=O(1)
$$

as $k \rightarrow \infty$, or

- for any $1 \leq i \leq N_{k}$ such that $d_{g}\left(x_{1, k}, x_{i, k}\right)=O\left(d_{k}\right)$

$$
\sup _{B_{\check{x}_{i, k}\left(\frac{1}{2}\right)}} \check{u}_{k} \rightarrow+\infty
$$

as $k \rightarrow \infty$.

Proof. Choose $k$ large enough and let $1 \leq i \leq N_{k}$ be such that $d_{g}\left(x_{1, k}, x_{i, k}\right)=O\left(d_{k}\right)$. We denote by $\check{x}_{i}$ the limit of $\left(\check{x}_{i, k}\right)_{k}$ in $\mathbb{R}^{n}$ when $k \rightarrow \infty$. Note that $\left|\check{x}_{i}\right|>0$ if $i \geq 2$. We first investigate separately each of the cases stated in Lemma 4.2. If (4.13) holds then by (4.8), (4.9) and standard elliptic theory, $\left(\check{u}_{k}\right)_{k}$ is uniformly bounded in $C^{1}\left(B_{\check{x}_{i, k}}\left(\frac{1}{4}\right)\right)$. By (4.1) we can thus find $\delta_{i}>0$ small enough and that does not depend on $k$ such that for $k$ large enough:

$$
\inf _{B_{\check{x}_{i, k}}\left(\delta_{i}\right)} \check{u}_{k} \geq \frac{1}{4}\left|\check{x}_{i}\right|^{1-\frac{n}{2}} .
$$

Assume on the contrary that (4.14) holds. By the definition of $d_{k}$ in (4.3), conditions (3.9) are satisfied with $x_{k}=x_{i, k}$ and $\rho_{k}=\frac{1}{8} d_{k}$ so Lemma 3.3 in the form of (3.39) applies and shows that:

$$
\check{u}_{k}\left(\check{x}_{i, k}\right) \rightarrow+\infty
$$

and that

$$
\check{u}_{k}\left(\check{x}_{i, k}\right)^{1-n+q_{k} \frac{n-2}{2}} \check{u}_{k}(x) \rightarrow \frac{\lambda_{i}}{\left|x-\check{x}_{i}\right|^{n-2}}+H_{i}(x)
$$

in $C_{\text {loc }}^{1}\left(B_{\check{x}_{i}}\left(\frac{1}{2}\right) \backslash\left\{\check{x}_{i}\right\}\right)$ as $k \rightarrow \infty$, where $\lambda_{i}>0$ and $H_{i}$ is a harmonic function in $B_{0}\left(\frac{5}{8}\right)$ satisfying $H_{i}\left(\check{x}_{i}\right) \leq 0$. Now we show that these two situations cannot simultaneously happen. Let $\mathcal{A} \subset$ $\left\{1 \ldots N_{k}\right\}$ be a finite collection of subscripts such that there exists a positive $R$ such that for all $i \in \mathcal{A}$

$$
d_{g}\left(x_{1, k}, x_{i, k}\right) \leq R d_{k} .
$$

Assume that there exist $i, j \in \mathcal{A}, i<j$, such that $x_{i, k}$ satisfies (4.13) and $x_{j, k}$ satisfies (4.14). Since $1-n+q_{k} \frac{n-2}{2} \rightarrow 1$ as $k \rightarrow \infty$, (4.16) and the $C^{1}$-convergence result (4.17) show that for any positive $r$ :

$$
\check{u}_{k} \rightarrow 0 \text { in } C_{l o c}^{1}\left(B_{\check{x}_{j}}(2 r) \backslash B_{\check{x}_{j}}(r)\right) .
$$

By their definition in (4.10), critical points $\check{x}_{l, k}$ satisfy for all $l, m \in \mathcal{A}, l \neq m,\left|\breve{x}_{l, k}-\breve{x}_{m, k}\right| \geq 1$. It is then possible to find a connected open set in $\mathbb{R}^{n}$, which we will call $U, U \subset B_{0}(R+1)$, that contains $B_{\check{x}_{i, k}}(1 / 4)$ and $B_{\breve{x}_{j, k}}(1 / 4)$ for any $k$ but that does not contain any other point $\check{x}_{l, k}$ for $l \neq i, j$. Let $0<r<\frac{1}{8}$ and consider

$$
V_{r, R}=U \backslash\left(\overline{B_{\check{x}_{i, k}}(r)} \cup \overline{B_{\check{x}_{j, k}}(r)}\right) \cap \Omega_{r, R+1} .
$$

By (4.12) we get

$$
\sup _{V_{r, R}} \check{u}_{k} \leq D_{r, R+1} \inf _{B_{\check{x}_{j, k}}(2 r) \backslash \bar{B}_{\check{x}_{j, k}}(r)} \check{u}_{k}
$$

so that using (4.18) we obtain that $\check{u}_{k}$ goes uniformly to 0 on every annulus centered at $\check{x}_{i, k}$, which contradicts (4.15). 
We are able to conclude the proof of Theorem 2.1. We first rule out the case (4.13). Assume by contradiction that, for any $1 \leq i \leq N_{k}$ such that $d_{g}\left(x_{1, k}, x_{i, k}\right)=O\left(d_{k}\right)$, 4.13 holds. By (4.12) and (4.13) the sequence $\left(\check{u}_{k}\right)_{k}$ is uniformly bounded in $L^{\infty}\left(B_{0}(R)\right)$ for all $R>0$. Using (4.8), (4.9) and standard elliptic theory there exists $\breve{u}$ satisfying

$$
\triangle_{\xi} \check{u}=f\left(x_{1}\right) \check{u}^{2^{*}-1}
$$

and such that

$$
\check{u}_{k} \rightarrow \check{u} \text { in } C_{l o c}^{1}\left(\mathbb{R}^{n}\right)
$$

as $k \rightarrow \infty$, where $x_{1}=\lim x_{1, k}$ up to a subsequence. We now show that $f\left(x_{1}\right)>0$. By (3.3) we therefore assume that $\left\|u_{k}\right\|_{H^{1}(M)} \leq C_{0}$. In particular the limit function $\check{u}$ belongs to $L^{2^{*}}\left(\mathbb{R}^{n}\right)$ since for any positive $R$ :

$$
\int_{B_{0}(R)} \check{u}_{k}^{2^{*}} d v_{\check{g}_{k}}=O\left(\int_{B_{x_{1}, k}\left(d_{k} R\right)} d_{k}^{\frac{2 q_{k}}{q_{k}-2}-n} u_{k}^{2^{*}} d v_{g}\right)=O\left(\int_{M} u_{k}^{2^{*}} d v_{g}\right)=O(1),
$$

where the last equality holds true because $\frac{2 q_{k}}{q_{k}-2}-n \geq 0$ and $d_{k} \rightarrow 0$. Passing to the limit (4.1) with (4.4) we obtain $\check{u}(0) \geq 1$. But $\check{u}$ cannot be subharmonic, non-zero and belong to $L^{2^{*}}\left(\mathbb{R}^{n}\right)$, hence $f\left(x_{1}\right)>0$. Moreover, we know that $\check{u}$ has at least two distinct critical points, 0 and $\check{x}_{2}$, since by assumption (4.4) there holds $\left|\breve{x}_{2, k}\right|=1$ for all $k$. This contradicts the classification result in Caffarelli-Gidas-Spruck [5] and thus contradicts [4.13). Hence for any $1 \leq i \leq N_{k}$ such that $d_{g}\left(x_{1, k}, x_{i, k}\right)=O\left(d_{k}\right)$ we have

$$
\check{u}_{k}\left(\check{x}_{i, k}\right) \rightarrow+\infty
$$

as $k \rightarrow \infty$. Proceeding as in the proof of Lemma 4.2 using the Harnack inequality (4.12) it is easily seen that $\check{u}_{k}$ blows up at the same speed at every concentration point at a finite distance from 0 : for any $1 \leq i \leq N_{k}$ such that $d_{g}\left(x_{1, k}, x_{i, k}\right)=O\left(d_{k}\right)$ there exist $\mu_{i}>0$ such that up to a subsequence

$$
\frac{\check{u}_{k}\left(\check{x}_{i, k}\right)}{\check{u}_{k}(0)} \rightarrow \mu_{i}
$$

as $k \rightarrow \infty$. Using (4.20), (4.17) shows that if $d_{g}\left(x_{1, k}, x_{i, k}\right)=O\left(d_{k}\right)$, then

$$
\check{u}_{k}(0)^{1-n+q_{k} \frac{n-2}{2}} \check{u}_{k}(x) \rightarrow \frac{\lambda_{i}}{\mu_{i}\left|x-\check{x}_{i}\right|^{n-2}}+\mu_{i}^{-1} H_{i}(x)
$$

in $C_{l o c}^{1}\left(B_{\breve{x}_{i}}\left(\frac{1}{2}\right) \backslash\left\{\check{x}_{i}\right\}\right)$ as $k \rightarrow \infty$. Let $R>0$. We know that $\left(N_{R, k}\right)_{k}$ and $\left(N_{2 R, k}\right)_{k}$ are bounded and we can thus assume they are constants $N_{R}, N_{2 R}$. Using Harnack's inequality (4.12) and (4.21) it is easily seen that

$$
\check{u}_{k}(0)^{1-n+q_{k} \frac{n-2}{2}} \check{u}_{k} \rightarrow \check{G} \text { in } C_{l o c}^{1}\left(B_{0}(R) \backslash\left\{\check{x}_{i}\right\}_{i=1, \cdots N_{2 R}}\right),
$$

where, by definition of $N_{R}$ as in (4.6), we can write that

$$
\check{G}(x)=\sum_{i=1}^{N_{R}} \frac{\lambda_{i}}{\mu_{i}\left|x-\check{x}_{i}\right|^{n-2}}+\check{H}(x),
$$

and where $\check{H}$ is a harmonic function in $\overline{B_{0}(R)}$. We write

$$
\check{G}(x)=\frac{\lambda_{1}}{|x|^{n-2}}+\left(\sum_{i=2}^{N_{R}} \frac{\lambda_{i}}{\mu_{i}\left|x-\check{x}_{i}\right|^{n-2}}+\check{H}(x)\right)
$$


and since $\check{u}_{k}(0) \rightarrow \infty$ as $k \rightarrow \infty$ we apply Lemma 3.3 with $x_{k}=x_{1, k}$ and $\rho_{k}=\frac{1}{16} d_{k}$ to obtain

$$
\sum_{i=2}^{N_{R}} \frac{\lambda_{i}}{\mu_{i}\left|\check{x}_{i}\right|^{n-2}}+\check{H}(0) \leq 0 .
$$

Independently, let $z_{0}$ be some point satisfying $\left|z_{0}\right|=R$ and $\left|z_{0}-\check{x}_{2}\right| \geq R-1$ and different from any $\check{x}_{i}, 3 \leq i \leq N_{2 R}$. Let $U_{0}$ be some connected open set in $\overline{B_{0}(R)}$ that contains 0 in its interior and $z_{0}$ on its boundary and avoids any other $\check{x}_{i}$. Then $\breve{G}-\lambda_{1}|x|^{2-n}-\frac{\lambda_{2}}{\mu_{2}}\left|x-\check{x}_{2}\right|^{2-n}$ is harmonic in $U_{0}$, so by the maximum principle:

$$
\begin{aligned}
\left(\check{G}-\frac{\lambda_{1}}{|x|^{n-2}}-\frac{\lambda_{2}}{\mu_{2}\left|x-\check{x}_{2}\right|^{n-2}}\right)(0) & \geq\left(\check{G}-\frac{\lambda_{1}}{|x|^{n-2}}-\frac{\lambda_{2}}{\mu_{2}\left|x-\check{x}_{2}\right|^{n-2}}\right)\left(z_{0}\right) \\
& \geq-\frac{\lambda_{1}}{R^{n-2}}-\frac{\lambda_{2}}{\mu_{2}(R-1)^{n-2}}
\end{aligned}
$$

since $\check{G}(x) \geq 0$ in $B_{0}(R) \backslash\{0\}$. With (4.23) and (4.25) we obtain in the end, since $\left|\check{x}_{2}\right|=1$ :

$$
\sum_{i=2}^{N_{R}} \frac{\lambda_{i}}{\mu_{i}\left|\check{x}_{i}\right|^{n-2}}+\check{H}(0) \geq \frac{\lambda_{2}}{\mu_{2}}-\frac{\lambda_{1}}{R^{n-2}}-\frac{\lambda_{2}}{\mu_{2}(R-1)^{n-2}},
$$

which contradicts (4.24) up to choosing $R$ large enough. This shows that (3.8) can never happen and concludes the proof of Theorem 2.1.

\section{A general MOUntain-PASS Situation.}

In this section we prove, as an application of Theorem 2.1] a result that states that equation (EL) has at least two solutions as soon as it has a mountain-pass structure. Starting with $E L$, we introduce the following equations for all $2 \leq q \leq 2^{*}$ :

$$
\triangle_{g} u+h u=f u^{q-1}+\frac{a}{u^{q+1}}
$$

and the associated energy functional, defined on $H^{1}(M)$ whenever the following expression makes sense (for instance for positive functions):

$$
I^{q}(u)=\frac{1}{2} \int_{M}\left(|\nabla u|_{g}^{2}+h u^{2}\right) d v_{g}-\frac{1}{q} \int_{M} f\left(u^{+}\right)^{q} d v_{g}+\frac{1}{q} \int_{M} \frac{a}{\left(u^{+}\right)^{q}} d v_{g} .
$$

Equation $\left.E L_{q}\right)$ is called subcritical if $q<2^{*}$ and critical if $q=2^{*}$. For any $2 \leq q \leq 2^{*}$ we call $S_{h, q}=S_{h, q}(M, g)$ the smallest positive constant in the embedding $H^{1}(M) \subset L^{q}(M)$ for the $H_{h}^{1}$-norm, i.e. satisfying:

$$
\|u\|_{L^{q}} \leq S_{h, q}^{\frac{1}{q}}\|u\|_{H_{h}^{1}},
$$

where the $H_{h}^{1}$-norm is as in (3.1). We have $S_{h, 2^{*}}=S_{h}$, where $S_{h}$ is as in (3.2). Following Hebey-Pacard-Pollack [12, to get rid of the negative exponent we consider for any $\varepsilon>0$ the perturbed functional on $H^{1}(M)$ :

$$
I_{\varepsilon}^{q}=\frac{1}{2} \int_{M}\left(|\nabla u|_{g}^{2}+h u^{2}\right) d v_{g}-\frac{1}{q} \int_{M} f\left(u^{+}\right)^{q} d v_{g}+\frac{1}{q} \int_{M} \frac{a}{\left(\varepsilon+\left(u^{+}\right)^{2}\right)^{q / 2}} d v_{g},
$$

where $2 \leq q \leq 2^{*}$. Our result states that, in low dimensions, each time the critical equation $(E L)$ has a mountain-pass structure each equation $\left.E L_{q}\right]$ with $2 \leq q \leq 2^{*}$ actually has two distinct positive solutions. We state it as follows. 
Theorem 5.1. Let $(M, g)$ be a n-dimensional closed Riemannian manifold with $3 \leq n \leq 5$ and $h, f$ and $a$ be smooth functions in $M$ such that $\triangle_{g}+h$ is coercive, $a \geq 0, a \neq 0$ and $\max _{M} f>0$. We assume that there exist two smooth positive functions $u_{0}$ and $u_{1}$ and two real numbers $\eta>0$ and $\rho>0$ such that $\left\|u_{1}-u_{0}\right\|_{H_{h}^{1}}<\rho$,

$$
I^{2^{*}}\left(u_{1}\right)<\eta
$$

where $I^{2^{*}}$ is as in (5.1), and

$$
\inf _{S} I_{\varepsilon}^{q}(u)>\eta
$$

for all $\varepsilon$ small enough and for all $q$ close enough to $2^{*}$, where we have let $S$ be the boundary set $S=\left\{u \in H^{1}(M),\left\|u-u_{0}\right\|_{H_{h}^{1}}=\rho\right\}$. Then for any $q$ close enough to $2^{*}$ equation EL admits two distinct smooth positive solutions.

Proof. We first consider the case $q<2^{*}$ and then prove the $q=2^{*}$ case with a stability argument. We thus assume that $2 \leq q<2^{*}$. The first solution is obtained as a limit of local minima of $I_{\varepsilon}^{q}$. The second one is obtained as a mountain-pass solution. On the closed ball

$$
B=\left\{u \in H^{1}(M),\left\|u-u_{0}\right\|_{H_{h}^{1}} \leq \rho\right\}
$$

the functional $I_{\varepsilon}^{q}$ is uniformly (in $\varepsilon$ ) bounded from below. Indeed, since $u_{1}$ is positive by Lebesgue's dominated convergence theorem we have:

$$
\lim _{q \rightarrow 2^{*}} \lim _{\varepsilon \rightarrow 0} I_{\varepsilon}^{q}\left(u_{1}\right)=I^{2^{*}}\left(u_{1}\right),
$$

so that using (5.4) there holds then for $\varepsilon$ small enough and for $q$ close to $2^{*}$,

$$
-\frac{1}{q} \max _{M}|f| S_{h, q}\left(\rho+\left\|u_{0}\right\|_{H_{h}^{1}}\right)^{q} \leq \inf _{B} I_{q}^{\varepsilon} \leq \eta
$$

where $S_{h, q}$ is as in (5.2) and $I_{q}^{\varepsilon}$ as in (5.3). Using Ekeland's variational principle we can let $\left(u_{k}\right)_{k}$ be a Palais-Smale minimising sequence for $I_{\varepsilon}^{q}$ such that $I_{\varepsilon}^{q}\left(u_{k}\right) \rightarrow \inf _{B} I_{\varepsilon}^{q}$ as $k \rightarrow \infty$ (see for instance Struwe [27], Chapter 1, Corollary 5.3 for the proof of this statement). The sequence $\left(u_{k}\right)_{k}$ thus satisfies

$$
\frac{1}{2} \int_{M}\left(\left|\nabla u_{k}\right|_{g}^{2}+h u_{k}^{2}\right) d v(g)-\frac{1}{q} \int_{M} f\left(u_{k}^{+}\right)^{q} d v(g)+\frac{1}{q} \int_{M} \frac{a}{\left(\varepsilon+\left(u_{k}^{+}\right)^{2}\right)^{\frac{q}{2}}}=\inf _{B} I_{\varepsilon}^{q}+o(1)
$$

and, for all $\Phi_{k} \in H^{1}(M)$,

$$
\begin{aligned}
\int_{M}\left\langle\nabla u_{k}, \nabla \Phi_{k}\right\rangle_{g}+h u_{k} \Phi_{k} d v(g) & -\int_{M} f\left(u_{k}^{+}\right)^{q-1} \Phi_{k} d v(g) \\
& -\int_{M} \frac{a u_{k}^{+}}{\left(\varepsilon+\left(u_{k}^{+}\right)^{2}\right)^{\frac{q}{2}+1}} \Phi_{k} d v(g)=o\left(\left\|\Phi_{k}\right\|_{H^{1}}\right) .
\end{aligned}
$$

Choosing $\Phi_{k}=u_{k}$ in (5.9) and combining with (5.8) yields, since $a \geq 0$ :

$$
\left(\frac{1}{2}-\frac{1}{q}\right) \int_{M} f\left(u_{k}^{+}\right)^{q} d v(g) \leqslant \inf _{B} I_{\varepsilon}^{q}+o\left(\left\|u_{k}\right\|_{H_{h}^{1}}\right)+o(1) .
$$

Using (5.10) in (5.8) we get for $k$ large enough:

$$
\left\|u_{k}\right\|_{H_{h}^{1}}^{2} \leq \frac{4 q}{q-2} \inf _{B} I_{\varepsilon}^{q}+o(1)
$$


and in particular $\inf _{B} I_{\varepsilon}^{q} \geq 0$. There exists then $u_{\varepsilon, q} \in H^{1}(M)$ such that, up to a subsequence, $u_{k} \rightarrow u_{\varepsilon, q}$ in $H^{1}(M)$. By standard integration theory $u_{\varepsilon, q}$ satisfies weakly

$$
\triangle_{g} u_{\varepsilon, q}+h u_{\varepsilon, q}=f\left(u_{\varepsilon, q}^{+}\right)^{q-1}+\frac{a\left(u_{\varepsilon, q}^{+}\right)}{\left(\varepsilon+\left(u_{\varepsilon, q}^{+}\right)^{2}\right)^{\frac{q}{2}+1}} .
$$

Multiplying (5.12) by $u_{\varepsilon, q}^{-}$and integrating it is easily seen that $u_{\varepsilon, q}$ is nonnegative almost everywhere. Since $a\left(\varepsilon+\left(u_{\varepsilon, q}^{+}\right)^{2}\right)^{-\frac{q}{2}-1}$ belongs to $L^{\infty}(M)$ standard bootstrap arguments show that $u_{\varepsilon, q}$ is smooth in $M$. Now we obtain a uniform bound from below on $u_{\varepsilon, q}$ and show that $\inf _{M} u_{\varepsilon, q}$ does not converge to 0 as $\varepsilon \rightarrow 0$. We consider for any positive $\delta$ the unique functions $\psi_{\delta}$ and $\psi_{0}$ solving

$$
\begin{aligned}
& \triangle_{g} \psi_{\delta}+h \psi_{\delta}=a-\delta f^{-}-\delta \\
& \triangle_{g} \psi_{0}+h \psi_{0}=a .
\end{aligned}
$$

Since $a \not \equiv 0, \psi_{0}>0$ in $M$. By standard elliptic theory $\psi_{\delta} \rightarrow \psi_{0}$ in $C^{0}(M)$ when $\delta \rightarrow 0$ and thus $\psi_{\delta_{0}}$ is positive for some $\delta_{0}>0$ small enough. Let $\varepsilon_{0}$ small enough so that $\inf _{M} \psi_{\delta_{0}} \varepsilon_{0}^{-2^{*}-2}>1$ and let $t_{0}>0$ small enough such that for any $q$ close enough to $2^{*}$

$$
\frac{\psi_{\delta_{0}}(x)}{\left(\varepsilon_{0}^{2}+t_{0}^{2} \psi_{\delta_{0}}(x)^{2}\right)^{\frac{q}{2}+1}}>1
$$

for any $x \in M$. Then for any $\varepsilon \leq \varepsilon_{0}$ and $t \leq t_{0}, \theta_{t}=t \psi_{\delta_{0}}$ satisfies

$$
\triangle_{g} \theta_{t}+h \theta_{t}<f \theta_{t}^{q-1}+\frac{a \theta_{t}}{\left(\varepsilon+\theta_{t}^{2}\right)^{\frac{q}{2}+1}} .
$$

Now we claim that there exists some $t>0$ small enough such that for any $\varepsilon$ small enough and any $q$ close enough to $2^{*}$ and for any smooth positive $\varphi$ solution of (5.12) there holds

$$
\varphi>\theta_{t}
$$

in $M$. This shows in particular that $u_{\varepsilon, q}$ is uniformly bounded from below in $\varepsilon$ and $q$. We prove the claim by contradiction and assume that for any positive $t$ there exists $\varepsilon_{t}>0,2 \leq q_{t}<2^{*}$, $\varphi$ a solution of (5.12) with $\varepsilon=\varepsilon_{t}$ and $q=q_{t}$ and $x_{t} \in M$ such that $\varphi\left(x_{t}\right) \leq \theta_{t}\left(x_{t}\right)$. Then, for some $\tilde{t} \in(0, t)$, and some $\tilde{x}_{t} \in M$,

$$
1=\inf _{M} \frac{\varphi}{\theta_{\tilde{t}}}=\frac{\varphi\left(\tilde{x}_{t}\right)}{\theta_{\tilde{t}}\left(\tilde{x}_{t}\right)} .
$$

In particular, with (5.16) we obtain that

$$
\theta_{\tilde{t}}\left(\tilde{x}_{t}\right)=\varphi\left(\tilde{x}_{t}\right) \text { and } \triangle_{g} \varphi\left(\tilde{x}_{t}\right) \leq \triangle_{g} \theta_{\tilde{t}}\left(\tilde{x}_{t}\right)
$$

which is impossible since $\theta_{\tilde{t}}$ is a strict subsolution of (5.12) by (5.14). This proves (5.15) which shows that $u_{\varepsilon, q}$ is positive for any $\varepsilon$ small enough and any $q$ close to $2^{*}$. Finally since $q<2^{*}$, $u_{k} \rightarrow u_{\varepsilon, q}$ in $L^{q}(M)$. Since $u_{\varepsilon, q}$ solves (5.12), a straightforward computation using (5.9) with $\Phi=u_{k}$ yields:

$$
\int_{M}\left|\nabla u_{k}-\nabla u_{\varepsilon, q}\right|_{g}^{2} d v_{g}=o(1)
$$

as $k \rightarrow \infty$ and so $u_{k} \rightarrow u_{\varepsilon, q}$ in $H^{1}(M)$. As a consequence $I_{\varepsilon}^{q}\left(u_{\varepsilon, q}\right)=\inf _{B} I_{\varepsilon}^{q}$. In particular by definition of $I_{\varepsilon}^{q}$ in (5.3) there holds $I_{\varepsilon}^{q}\left(u_{1}\right) \leq I^{q}\left(u_{1}\right)$, where $I^{q}$ is as in (5.1). Since $u_{1} \in B$, with $B$ as in (5.6), using (5.4) there holds, for $q$ close enough to $2^{*}$ :

$$
I_{\varepsilon}^{q}\left(u_{\varepsilon, q}\right) \leq I^{q}\left(u_{1}\right)<\eta .
$$

Now we let $\varepsilon \rightarrow 0$. By (5.11) and (5.7) there exists a nonnegative $u_{q} \in H^{1}(M)$ such that $u_{\varepsilon, q}$ converges, up to a subsequence, weakly to $u_{q}$ in $H^{1}(M)$. With (5.15) and standard integration 
theory we can pass to the limit in (5.12) and get that $u_{q}$ is a smooth solution of EL $E L_{q}$, once again positive by (5.15). Here again, since $q<2^{*}$, a similar computation to the one in (5.17) shows that

$$
\int_{M}\left|\nabla u_{\varepsilon, q}-\nabla u_{q}\right|^{2} d v_{g}=o(1)
$$

as $\varepsilon \rightarrow 0$, so $u_{\varepsilon, q} \rightarrow u_{q}$ strongly in $H^{1}(M)$. In particular (5.15) and Lebesgue's dominated convergence theorem show that $I_{\varepsilon}^{q}\left(u_{\varepsilon, q}\right) \rightarrow I^{q}\left(u_{q}\right)$, where $I^{q}$ is as in (5.1). By (5.18) this gives, for $q$ close enough to $2^{*}$ :

$$
I^{q}\left(u_{q}\right) \leq I^{q}\left(u_{1}\right)<\eta .
$$

Now we take into account the mountain-pass structure of $I_{\varepsilon}^{q}$ in order to construct a second solution. Since $\max _{M} f>0$ it is possible to find a smooth positive function $\psi$ in $M$ such that

$$
\int_{M} f \psi^{2^{*}} d v_{g}>0
$$

Then by (5.1) and (5.20), $I^{2^{*}}(t \psi) \rightarrow-\infty$ as $t$ goes to infinity. We can thus pick a large real number $T$ such that, for any $q$ close enough to $2^{*}$ and for any positive $\varepsilon$ small enough,

$$
I_{\varepsilon}^{q}(T \psi)<\eta
$$

and also $\left\|T \psi-u_{0}\right\|_{H_{h}^{1}}>\rho$, where $\eta, u_{0}$ and $\rho$ are as in the statement of Theorem 5.1. On the other side by assumption (5.5) we get for any $q$ close enough to $2^{*}$ and for any positive $\varepsilon$ small enough

$$
I_{\varepsilon}^{q}(u)>\eta
$$

for all $u \in S=\left\{u \in H^{1}(M),\left\|u-u_{0}\right\|_{H_{h}^{1}}=\rho\right\}$. Thus with (5.18), (5.21) and (5.22), for any $q$ close enough to $2^{*}$, and for any $\varepsilon$ small enough, we can apply the mountain-pass lemma as stated in Rabinowitz 22 that provides us with a Palais-Smale sequence $\left(v_{k}\right)_{k}$ such that

$$
I_{\varepsilon}^{q}\left(v_{k}\right) \rightarrow c_{\varepsilon, q}
$$

as $k \rightarrow+\infty$, where we have set

$$
c_{\varepsilon, q}=\inf _{h \in \Gamma} \max _{u \in h([0 ; 1])} I_{\varepsilon}^{q}(u)
$$

and where $\Gamma$ is the set of paths $h:[0,1] \rightarrow H^{1}(M)$ such that $h(0)=u_{\varepsilon, q}$ and $h(1)=T \psi$. Using (5.5) and considering as a special $h$ a parametrization of the segment $\left[u_{\varepsilon, q} ; T \psi\right]$ it is easily seen, using (5.11) and (5.15) that there exists a positive $C_{0}$ such that

$$
\eta \leq c_{\varepsilon, q} \leq C_{0}
$$

for $\varepsilon$ small enough and for $q$ close to $2^{*}$. Therefore the same computation that led to (5.11) works again and shows that

$$
\left\|v_{k}\right\|_{H_{h}^{1}} \leq \frac{4 q}{q-2} C_{0}+1
$$

with $C_{0}$ as in (5.25). With (5.26) and standard integration theory, mimicking the proof of (5.17) one shows that the sequence $\left(v_{k}\right)_{k}$ converges strongly in $H^{1}(M)$ to some nonnegative function $v_{\varepsilon, q}$ that solves (5.12). Passing in (5.22) to the limit as $k \rightarrow \infty$ shows that

$$
I_{\varepsilon}^{q}\left(v_{\varepsilon, q}\right)=c_{\varepsilon, q} \geq \eta
$$

by (5.25). Also with (5.15) we get that $v_{\varepsilon, q}$ is uniformly bounded from below in $\varepsilon$ and in $q$. Thus repeating the whole argument once again it is easily seen that $v_{\varepsilon, q}$ converges strongly in $H^{1}(M)$ 
as $\varepsilon \rightarrow 0$ to some smooth positive solution $v_{q}$ of $E L_{q}$. With (5.15) we can pass in (5.27) to the limit as $\varepsilon \rightarrow 0$ and obtain:

$$
I^{q}\left(v_{q}\right) \geq \eta .
$$

In particular, (5.19) and (5.28) show that $u_{q} \neq v_{q}$. This shows Theorem 5.1 for all $2 \leq q<2^{*}$ and in any dimension. We now conclude the proof and show that there still exist two different solutions of the critical equation in low dimensions, that is when $3 \leq n \leq 5$. By (5.11) and (5.26) the sequences $\left(u_{q}\right)_{q}$ and $\left(v_{q}\right)_{q}$ are bounded in $H^{1}(M)$. Since $\triangle_{g}+h$ is coercive and $\max _{M} f>0$ we can let $\left(q_{k}\right)_{k}$ be some sequence converging to $2^{*}$ and apply Theorem 2.1] with $a_{k} \equiv a$ to the sequences $\left(u_{q_{k}}\right)_{k}$ and $\left(v_{q_{k}}\right)_{k}$. There thus exist $u$ and $v$ two smooth positive functions that solve (EL) such that $u_{q_{k}} \rightarrow u$ and $v_{q_{k}} \rightarrow v$ in $C^{1, \alpha}(M)$ for all $0<\alpha<1$. Passing (5.19) and (5.28) to the limit as $k \rightarrow \infty$ finally gives:

$$
I^{2^{*}}(u) \leq I^{2^{*}}\left(u_{1}\right)<\eta \leq I^{2^{*}}(v)
$$

which shows that $u$ and $v$ are distinct.

Note that the construction of $u$ and $v$ is consistent with the computation of the degree of (EL) we performed in (2.1). Indeed, $u$ is obtained as a limit of local minima, hence of solutions of index 0 while $v$ is a mountain-pass solution, hence (generically) of index +1 .

\section{A minimal solution of (EL).}

We now investigate more precisely the influence of the parameter $a$ and consider the following equation:

$$
\triangle_{g} u+h u=f u^{2^{*}-1}+\frac{a}{u^{2^{*}+1}}
$$

where $h, f$ and $a$ are smooth functions in $M, \triangle_{g}+h$ is coercive, $\max _{M} f>0$ and $a$ is nonnegative and nonzero. Using the sub and super solution method we show that each time equation $E L_{a}$ has a smooth positive solution it has a smallest solution for the $L^{\infty}$-norm:

Proposition 6.1. Let $(M, g)$ be a closed Riemannian manifold of dimension $n \geq 3$ and a be a non zero smooth function in $M$. Let $h$ and $f$ be smooth functions in $M$ such that $\triangle_{g}+h$ is coercive and $\max _{M} f>0$ and $a \geq 0$. Assume that $E L_{a}$ has a smooth positive solution. Then there exists a smooth positive function $\varphi(a)$ solving $E L_{a}$ such that for any other solution $\varphi$ of $E L_{a}$ with $\varphi \not \equiv \varphi(a)$ there holds $\varphi>\varphi(a)$. Moreover $\varphi(a)$ is stable, in the sense that for any $\psi \in H^{1}(M)$ there holds

$$
\int_{M}|\nabla \psi|^{2}+\left[h-\left(2^{*}-1\right) f \varphi(a)^{2^{*}-2}+\left(2^{*}+1\right) \frac{a}{\varphi(a)^{2^{*}+2}}\right] \psi^{2} d v_{g} \geq 0,
$$

and the mapping $a \mapsto \varphi(a)$ in nondecreasing in the following sense: if $a_{1} \leq a_{2}$ in $M$, provided $\varphi\left(a_{1}\right)$ and $\varphi\left(a_{2}\right)$ exist, there holds $\varphi\left(a_{1}\right) \leq \varphi\left(a_{2}\right)$.

Proof. Let $a \geq 0$ be a nonzero smooth function such that $E L_{a}$ has a solution. Mimicking the proof of (5.15) we start proving that there exists a positive number that bounds from below all the solutions of $\left.E L_{a}\right)$. As in (5.15), notice that there always exist sub-solutions of $\left.E L_{a}\right)$ as small as we want. Indeed, for any $\delta \geq 0$ we let $u_{\delta}$ be the unique solution of

$$
\triangle_{g} u_{\delta}+h u_{\delta}=a-\delta f^{-}-\delta,
$$

where $f^{-}=-\min (f, 0)$. Since $a$ is nonnegative and nonzero, the maximum principle shows that $u_{0}>0$ in $M$. By standard elliptic theory $\left\|u_{\delta}-u_{0}\right\|_{\infty} \rightarrow 0$ as $\delta$ goes to 0 so for some $\delta_{0}>0$ small enough we have $u_{\delta_{0}}>0$. Then for $\varepsilon$ small enough,

$$
v_{\varepsilon}=\varepsilon u_{\delta_{0}}
$$


is a strict sub-solution of $E L_{a}$ since, by (6.1),

$$
\triangle_{g} v_{\varepsilon}+h v_{\varepsilon}=\varepsilon a-\varepsilon \delta_{0} f^{-}-\varepsilon \delta_{0}<\frac{a}{v_{\varepsilon}^{2 *+1}}+f v_{\varepsilon}^{2^{*}-1} .
$$

Now we claim that there exists some $\varepsilon_{0}>0$ such that for any positive solution $\varphi$ of $E L_{a}$ there holds

$$
\varphi>v_{\varepsilon_{0}}
$$

in $M$, where $v_{\varepsilon_{0}}$ is as in (6.2). We prove the claim by contradiction and assume that there exists $\varphi_{\varepsilon}$ solution of $\left[E L_{a}\right]$, and $x_{\varepsilon} \in M$, such that $\varphi_{\varepsilon}\left(x_{\varepsilon}\right) \leq v_{\varepsilon}\left(x_{\varepsilon}\right)$ for all $\varepsilon>0$. Then, for some $\tilde{\varepsilon} \in(0, \varepsilon)$, and some $\tilde{x}_{\varepsilon} \in M$,

In particular, we obtain that

$$
1=\inf _{M} \frac{\varphi_{\varepsilon}}{v_{\tilde{\varepsilon}}}=\frac{\varphi_{\varepsilon}\left(\tilde{x}_{\varepsilon}\right)}{v_{\tilde{\varepsilon}}\left(\tilde{x}_{\varepsilon}\right)}
$$

$$
v_{\tilde{\varepsilon}}\left(\tilde{x}_{\varepsilon}\right)=\varphi_{\varepsilon}\left(\tilde{x}_{\varepsilon}\right) \text { and } \triangle_{g} \varphi_{\varepsilon}\left(\tilde{x}_{\varepsilon}\right) \leq \triangle_{g} v_{\tilde{\varepsilon}}\left(\tilde{x}_{\varepsilon}\right)
$$

which is impossible since $v_{\tilde{\varepsilon}}$ is a strict subsolution of $E L_{a}$. Now we prove the existence of a minimal solution of $E L_{a}$. We follow here the arguments in Sattinger 24. For $x \in M$ and $u>0$ we let

$$
F(x, u)=f(x) u(x)^{2^{*}-1}+\frac{a(x)}{u(x)^{2^{*}+1}}-h(x) u(x) .
$$

Let $\psi$ be a solution of $E L_{a}$ and let $w$ be a strict subsolution of $E L_{a}$ which is less than any positive solution of $\left.E L_{a}\right)$. We proved the existence of such a $w$ in $[6.3)$. Also we let $K>0$ be large enough such that for any $x \in M$, and any $\min _{M} w \leq u \leq \max _{M} \psi$,

$$
F(x, u)+K u \geq 0 \text { and } \frac{\partial F}{\partial u}(x, u)+K \geq 0 .
$$

For any smooth positive function $u$, we define $T u$ as the unique solution of

$$
\triangle_{g} T u+K T u=F(\cdot, u)+K u .
$$

As a first remark, for any two positive functions $u$ and $v$ in the range

$$
\min _{M} w \leq u, v \leq \max _{M} \psi
$$

we have:

$$
\left(\triangle_{g}+K\right)(T u-T v)(x)=F(x, u)-F(x, v)+K(u(x)-v(x)) .
$$

Then, by the strong maximum principle, we obtain that

$$
T u<T v \text { as long as } u \leq v \text { and } u \not \equiv v .
$$

The iterative sub and super solution method applied in the range $w \leq \varphi \leq \psi$ and starting from the strict sub-solution $w$ provides a sequence $v_{n}=T^{n} w$ which is non decreasing by the maximum principle and converges to a fixed-point of $T$, that is to say a solution of $E L_{a}$ ) (see 24 for more details). We shall call this solution $\varphi(a)$ :

$$
\varphi(a)=\lim _{n \rightarrow \infty} T^{n} w
$$

By standard elliptic theory, $\varphi(a)$ is smooth. Note in passing that all the above arguments still work if we only assume that $a$ is continuous, but in this case $\varphi(a)$ constructed as in (6.7) will only be of class $C^{1, \alpha}$ for any $0<\alpha<1$. Now we show that $\varphi(a)$ does not depend on $\psi$ and on $w$. First, $\varphi(a)$ as in (6.7) does not depend on $\psi$. We let $\psi_{1}$ and $\psi_{2}$ be two solutions of $E L_{a}$. We let $K_{i}$, $i=1,2$ be positive constants satisfying (6.4) in $\left[\min _{M} w ; \max _{M} \psi_{i}\right], T_{i}$ be the operator defined as in (6.5) and $\varphi_{i}$ the associated solution as in (6.7). Since $\left(T_{1}^{n} w\right)$ is non decreasing there holds $\varphi_{1} \geq w$. If we assume for instance that $\max _{M} \psi_{1} \leq \max _{M} \psi_{2}$ then $\varphi_{1} \in\left[\min _{M} w ; \max _{M} \psi_{2}\right]$ and 
thus, by (6.7) and the maximum principle there holds $\varphi_{2} \leq \varphi_{1}$ since $T_{2}\left(\varphi_{1}\right)=\varphi_{1}$. But then $\varphi_{2}$ is a solution of $E L_{a}$ with $\min _{M} w \leq \varphi_{2} \leq \max _{M} \psi_{1}$ and thus, once again by the maximum principle, $\varphi_{1} \leq \varphi_{2}$. This proves that $\varphi(a)$ does not depend on $\psi$. Now we prove that $\varphi(a)$ does not depend on the strict subsolution $w$, provided that $w$ is less than any positive solution of $E L_{a}$. Indeed, for any $\psi$ solution of ELa , if $w_{1}$ and $w_{2}$ are two such subsolutions, and $\varphi_{1}$ and $\varphi_{2}$ are the associated solutions as in (6.7), there holds $w_{1} \leq \varphi_{2}$ and $w_{2} \leq \varphi_{1}$. We conclude once again with the maximum principle that $\varphi_{1} \leq \varphi_{2}$ and $\varphi_{2} \leq \varphi_{1}$. By the definition of $\varphi(a)$ in (6.7), and what we just proved, for any $\psi$ solution of $\left.E L_{a}\right)$ there holds that $w<\varphi(a) \leq \psi$, where $w$ is a subsolution that is less than any solution of $\left.E L_{a}\right)$. With (6.6) we obtain the desired property:

$$
\varphi(a)<\psi \text { or } \varphi(a) \equiv \psi \text {. }
$$

The stability of $\varphi(a)$ is a consequence of the minimality of $\varphi(a)$. We denote by $\lambda_{0}$ the first eigenvalue of the linearized operator of equation $E L_{a}$ at $\varphi(a)$. The stability of $\varphi(a)$ as stated in Proposition 6.1 amounts to say that $\lambda_{0} \geq 0$. Assume by contradiction that $\lambda_{0}<0$ and denote by $\psi_{0}$ the associated positive eigenvector. Let $w$ be a subsolution that is less than any solution of $E L_{a}$. Let $\varphi_{\delta}=\varphi(a)-\delta \psi_{0}$ for any positive $\delta$. For $\delta>0$ small enough one has

$$
w<\varphi_{\delta}<\varphi(a)
$$

and a straightforward calculation shows that

$$
\triangle_{g} \varphi_{\delta}+h \varphi_{\delta}-f \varphi_{\delta}^{2^{*}-1}-\frac{a}{\varphi_{\delta}^{2^{*}+1}}=-\delta \lambda_{0} \psi_{0}+o(\delta)>0
$$

so that $\varphi_{\delta}$ is a strict supersolution of $E L_{a}$ satisfying $w<\varphi_{\delta}<\varphi(a)$ for $\delta$ small enough. By the iterative sub and super solution method we then get a solution $\psi$ of $\left(E L_{a}\right)$ such that $w<\psi<\varphi_{\delta}$, and this is in contradiction with (6.8). Finally, if $a_{1} \leq a_{2}$ are nonnegative nonzero functions on $M, \varphi\left(a_{2}\right)$ is a super solution of equation $E L_{a}$ with $a=a_{1}$. By the minimality of $\varphi\left(a_{1}\right)$ we then have $\varphi\left(a_{1}\right) \leq \varphi\left(a_{2}\right)$.

\section{Multiplicity of solutions of EL}

Let $(M, g)$ be a closed $n$-dimensional Riemannian manifold, $3 \leq n \leq 5$, and $h, f, a$ be smooth functions in $M$ with $a$ nonzero and nonnegative, $\triangle_{g}+h$ coercive and $\max _{M} f>0$. Using Theorem 5.1 and Proposition 6.1 we conclude in this section the proof of Theorem 1.1 Remember that we are investigating the number of solutions of the following equation:

$$
\triangle_{g} u+h u=f u^{2^{*}-1}+\frac{\theta a}{u^{2^{*}+1}},
$$

according to the value of the positive parameter $\theta$.

7.1. Two solutions when $a$ is small. We now prove the existence of $\theta_{1}$ as in the statement of Theorem 1.1. Following Hebey-Pacard-Pollack [12] we prove that if there exists a positive function $\varphi$ in $M$ satisfying $\|\varphi\|_{H_{h}^{1}}=1$ and

$$
\int_{M} \frac{a}{\varphi^{2^{*}}} d v_{g} \leqslant \frac{C(n)}{\left(S_{h} \max _{M}|f|\right)^{n-1}}
$$

where

$$
C(n)=\frac{1}{n-2} \frac{1}{(2(n-1))^{\frac{2^{*}}{2}}},
$$


then (EL) has at least two smooth positive solutions. Note that for the sake of simplicity in (17.1) we have let $S_{h}=S_{h, 2^{*}}$, where $S_{h, 2^{*}}$ is as in (5.2). To do this, we prove that if (7.1) is satisfied so are the assumptions of Theorem 5.1. We introduce for any $2 \leq q \leq 2^{*}$ the function

$$
\Phi_{q}(t)=\frac{1}{2} t^{2}-\frac{\max _{M}|f|}{q} S_{h, q} t^{q}
$$

defined on $\mathbb{R}^{+}$which attains its maximum at

$$
t_{0, q}=\left(\frac{1}{S_{h, q} \max _{M}|f|}\right)^{\frac{1}{q-2}}
$$

where $S_{h, q}$ is as in (5.2). The value of $\Phi_{q}$ at its maximum is, for $2 \leq q \leq 2^{*}$, given by

$$
\Phi_{q}\left(t_{0, q}\right)=\left(\frac{1}{2}-\frac{1}{q}\right) \frac{1}{\left(S_{h, q} \max _{M}|f|\right)^{\frac{2}{q-2}}} .
$$

In particular, since $S_{h, q} \rightarrow S_{h, 2^{*}}$ as $q \rightarrow 2^{*}$ :

$$
\Phi_{q}\left(t_{0, q}\right) \rightarrow \Phi_{2^{*}}\left(t_{0,2^{*}}\right)>0
$$

as $q \rightarrow 2^{*}$. We shall write for the sake of simplicity $\Phi\left(t_{0}\right)=\Phi_{2^{*}}\left(t_{0,2^{*}}\right)$ in the following, where we have thanks to (7.4):

$$
\Phi\left(t_{0}\right)=\frac{1}{n}\left(S_{h} \max _{M}|f|\right)^{-\frac{n-2}{2}} .
$$

By their definition in (5.3), the perturbed functionals $I_{\varepsilon}^{q}$ satisfy for any $u \in H^{1}(M)$ :

$$
I_{\varepsilon}^{q}(u) \geq \Phi_{q}\left(\|u\|_{H_{1}^{h}}\right) .
$$

In particular, from (7.7) there holds for any $2 \leq q \leq 2^{*}$, any $\varepsilon$ and any $\|u\|_{H_{h}^{1}}=t_{0, q}$ :

$$
I_{\varepsilon}^{q}(u) \geq \Phi_{q}\left(t_{0, q}\right) \text {. }
$$

We now let

$$
t_{1}=(2(n-1))^{-\frac{1}{2}} t_{0} .
$$

As one can easily check thanks to (7.3), (7.6) and (7.9) there holds:

$$
\frac{1}{2} t_{1}^{2}+\frac{\max _{M}|f|}{2^{*}} S_{h} t_{1}^{2^{*}}<\frac{1}{2} \Phi\left(t_{0}\right)
$$

Let $\varphi$ be as in (7.1) with $\|\varphi\|_{H_{h}^{1}}=1$. By (7.9) there holds $\left\|t_{1} \varphi\right\|_{H_{h}^{1}}<t_{0}$. From the definition of $I^{2^{*}}$ as in (5.1) and by (7.1) there holds:

$$
I^{2^{*}}\left(t_{1} \varphi\right) \leq \frac{1}{2} t_{1}^{2}+\frac{\max _{M}|f|}{2^{*}} S_{h} t_{1}^{2^{*}}+\frac{1}{2^{*}} t_{1}^{-2^{*}} \frac{C(n)}{\left(S_{h} \max _{M}|f|\right)^{n-1}}
$$

which becomes, with (7.2), (7.9) and (17.10):

$$
I^{2^{*}}\left(t_{1} \varphi\right)<\frac{1}{2} \Phi\left(t_{0}\right)+\frac{1}{2 n}\left(S_{h} \max _{M}|f|\right)^{-\frac{n-2}{2}}<\Phi_{0}\left(t_{0}\right) .
$$

By (7.5) and with (7.8) and (7.12) we can apply Theorem 5.1 and obtain two solutions of (EL) for $3 \leq n \leq 5$. As one can check, condition (17.1) is satisfied when $\varphi$ is a positive constant equal to $\int_{M} h d v_{g}$ whenever $a$ satisfies

$$
\|a\|_{\infty}<C(n)\left(S_{h} \max _{M}|f|\right)^{1-n} V_{g}^{-1}\left(\int_{M} h d v_{g}\right)^{-\frac{2^{*}}{2}},
$$


where $V_{g}$ is the volume of $(M, g)$. We have thus just proved that for $\theta$ small enough, equation $E L_{\theta}$ has two smooth positive solutions. Thus, the parameter $\theta_{1}$ appearing in the statement of Theorem 1.1 can be defined as

(7.13) $\theta_{1}=\sup \left\{\xi\right.$ such that for any $\theta \in[0, \xi]$ there exist at least two solutions of $\left[E L_{\theta} D\right\}$ and (7.1) provides us with a lower bound on $\theta_{1}$ :

$$
\theta_{1} \geq \inf \frac{C(n)}{\left(\max _{M}|f| S_{h}\right)^{n-1}}\left(\int_{M} \frac{a}{\varphi^{2^{*}}} d v(g)\right)^{-1},
$$

where the infimum is taken over all the smooth positive function $\varphi$ on $M$ with $\|\varphi\|_{H_{h}^{1}}=1$ and $C(n)$ is as in (7.2).

7.2. The Intermediate case. Just as we defined $\theta_{1}$ in (7.13), we let:

$$
\left.\theta_{2}=\sup \left\{\theta>0 \text { such that equation } E L_{\theta}\right] \text { admits a smooth positive solution }\right\} \text {. }
$$

There holds $\theta_{1} \leq \theta_{2}$ and for any $\theta<\theta_{2}$ there exists at least one solution of $\left(E L_{\theta} \mid\right.$. Indeed, by definition of $\theta_{2}$ in (7.14), for any $\theta<\theta_{2}$ there exists $\theta<\theta_{0} \leq \theta_{2}$ such that a solution $u_{\theta_{0}}$ of $E L_{\theta}$ for $\theta=\theta_{0}$ exists. For any $\xi<\theta_{0}, u_{\theta_{0}}$ is then a super solution of

$$
\triangle_{g} u+h u=f u^{2^{*}-1}+\frac{\xi a}{u^{2^{*}+1}} .
$$

Since there exist sub solutions of $\left(E L_{\xi}\right)$ as small as we want by $[6.2], E L_{\xi}$ has at least one smooth positive solution.

7.3. The Positive case. It is known that $\theta_{2}$ is finite when $f>0$, see Theorem 2.1 in HebeyPacard-Pollack [12. To conclude the proof of Theorem 1.1] we now show, when $f>0$, that for every $\theta<\theta_{2}$ equation $\left.E L_{\theta}\right]$ has at least two distinct solutions and that for $\theta=\theta_{2}$ there is exactly one solution. We define for positive $u \in H^{1}(M)$ the energy functional of $\left.E L_{\theta}\right]$ :

$$
I_{\theta}(u)=\frac{1}{2} \int_{M}\left(|\nabla u|_{g}^{2}+h u^{2}\right) d v_{g}-\frac{1}{2^{*}} \int_{M} f u^{2^{*}} d v_{g}+\frac{\theta}{2^{*}} \int_{M} \frac{a}{u^{2^{*}}} d v_{g} .
$$

For any $\theta<\theta_{2}$, Proposition 6.1 provides us with a canonical minimal solution which we shall call $\varphi_{\theta}$. It is stable, that is to say that $D^{2} I_{\theta}\left(\varphi_{\theta}\right)$ is a nonnegative bilinear form. Now we investigate the path $\theta \mapsto \varphi_{\theta}$ and show that $D^{2} I_{\theta}\left(\varphi_{\theta}\right)$ is actually a definite positive bilinear form if $\theta<\theta_{2}$. This will give us a suitable mountain-pass structure to apply Theorem[5.1. Proposition 6.1] shows that the path $\theta \mapsto \varphi_{\theta}$ is increasing: $\varphi_{\theta}(x)<\varphi_{\eta}(x)$ for all $x \in M$ and $\theta<\eta$. We first prove that $\theta \mapsto \varphi_{\theta}$ is continuous:

Lemma 7.1. The path $\theta \mapsto \varphi_{\theta}$ is continuous for the $L^{\infty}(M)$-norm, that is to say:

$$
\left\|\varphi_{\theta_{0}}-\varphi_{\theta}\right\|_{\infty} \rightarrow 0
$$

as $\theta \rightarrow \theta_{0}$, for any $0<\theta_{0}<\theta_{2}$, where $\theta_{2}$ is as in (7.14).

Proof. Let $\left(\theta_{k}\right)_{k}$ be a sequence of positive numbers converging to some $0<\theta_{0}<\theta_{2}$; up to assuming $k$ large enough, we can assume that $0<\theta_{0}-\varepsilon \leq \theta_{k} \leq \theta_{0}+\varepsilon<\theta_{2}$ for all $k$, for some positive $\varepsilon<\left(\theta_{2}-\theta_{0}\right) / 2$. By Proposition 6.1 we have

$$
\varphi_{\theta_{0}-\varepsilon} \leq \varphi_{\theta_{k}} \leq \varphi_{\theta_{0}+\varepsilon},
$$

where $\varphi_{\theta_{k}}$ denotes the minimal solution of $\left[E L_{\theta}\right.$ for $\theta=\theta_{k}$. By standard elliptic theory and (7.17) there exists $\varphi_{0}$ in $C^{2}(M)$ solution of

$$
\triangle_{g} \varphi_{0}+h \varphi_{0}=f \varphi_{0}^{2^{*}-1}+\theta_{0} a \varphi_{0}^{-2^{*}-1}
$$


such that $\varphi_{\theta_{k}} \rightarrow \varphi_{0}$ in $C^{2}(M)$ as $k \rightarrow \infty$. By definition of the minimal solution as given by Proposition 6.1. there holds $\varphi_{0} \geq \varphi_{\theta_{0}}$. We proceed by contradiction and assume that $\varphi_{\theta_{0}}<\varphi_{0}$. We then define for any $t \in[0 ; 1]$

$$
m(t)=I_{\theta_{0}}\left(t \varphi_{\theta_{0}}+(1-t) \varphi_{0}\right),
$$

where $I_{\theta_{0}}$ is as in (7.15). By proposition 6.1. for any $k, \varphi_{\theta_{k}}$ is stable, that is $D^{2} I_{\theta_{k}}\left(\varphi_{\theta_{k}}\right) \geq 0$, and thus $\varphi_{0}$ is stable. Hence $m^{\prime \prime}(0) \geq 0$. Using (17.15) we can compute $m^{(3)}(t)$ for any $t \in[0,1]$, where $m^{(3)}$ is the third derivative of $m$. There holds

$$
\begin{array}{r}
m^{(3)}(t)=-\left(2^{*}-1\right)\left(2^{*}-2\right) \int_{M} f\left(t \varphi_{\theta_{0}}+(1-t) \varphi_{0}\right)^{2^{*}-3}\left(\varphi_{\theta_{0}}-\varphi_{0}\right)^{3} d v_{g} \\
-\left(2^{*}+1\right)\left(2^{*}+2\right) \int_{M} \theta_{0} a\left(t \varphi_{\theta_{0}}+(1-t) \varphi_{0}\right)^{-2^{*}-3}\left(\varphi_{\theta_{0}}-\varphi_{0}\right)^{3} d v_{g}
\end{array}
$$

Since $\varphi_{0}>\varphi_{\theta_{0}}$ and $f>0, m^{(3)}(t)$ is positive for all $t \in(0,1)$. Hence $m^{\prime \prime}$ is a positive function of $t$ for $0<t \leq 1$ and $m^{\prime}$ is increasing in $(0,1)$. But this is impossible since both $\varphi_{0}$ and $\varphi_{\theta_{0}}$ are solutions of (7.16) and there thus holds $m^{\prime}(0)=m^{\prime}(1)=0$. Hence $\varphi_{0}=\varphi_{\theta_{0}}$ and $\theta \mapsto \varphi_{\theta}$ is continuous.

For any $\theta, \eta<\theta_{2}$ we have:

$$
\begin{array}{r}
\triangle_{g}\left(\varphi_{\theta}-\varphi_{\eta}\right)+h\left(\varphi_{\theta}-\varphi_{\eta}\right)=f\left(\varphi_{\theta}^{2^{*}-1}-\varphi_{\eta}^{2^{*}-1}\right)+a \theta\left(\frac{1}{\varphi_{\theta}^{2^{*}+1}}-\frac{1}{\varphi_{\eta}^{2^{*}+1}}\right) \\
+(\theta-\eta) \frac{a}{\varphi_{\eta}^{2^{*}+1}}
\end{array}
$$

After multiplication of (7.18) by $\varphi_{\theta}-\varphi_{\eta}$ and integration, using Lemma 7.1 and since $\triangle_{g}+h$ is coercive we get that $\theta \mapsto \varphi_{\theta}$ is continuous for the $H^{1}$ norm. We let $\lambda(\theta)$ be the first eigenvalue of the linearized operator at $\varphi_{\theta}$ and $u_{\theta}$ be the positive associated eigenvector with $\left\|u_{\theta}\right\|_{\infty}=1$, thus satisfying:

$$
\triangle_{g} u_{\theta}+h u_{\theta}-\left(2^{*}-1\right) f \varphi_{\theta}^{2^{*}-2} u_{\theta}+\left(2^{*}+1\right) \frac{\theta a}{\varphi_{\theta}^{2^{*}+2}} u_{\theta}=\lambda(\theta) u_{\theta}
$$

To prove that $D^{2} I_{\theta}\left(\varphi_{\theta}\right)$ is positive-definite for any $\theta$ we proceed by contradiction and assume that for some $0<\theta_{0}<\theta_{2}$ there holds $\lambda\left(\theta_{0}\right)=0$. We let $\varphi_{0}=\varphi_{\theta_{0}}$ and $u_{0}=u_{\theta_{0}}$. Our goal is to obtain an asymptotic expansion of $\varphi_{\theta}$ in $H^{1}(M)$. First, we claim that there holds:

$$
\frac{\left\|\varphi_{\theta}-\varphi_{0}\right\|_{H_{h}^{1}}}{\theta-\theta_{0}} \rightarrow+\infty
$$

as $\theta \rightarrow \theta_{0}$. Indeed, we define for all $\theta \neq \theta_{0}$ :

$$
\psi_{\theta}=\frac{\varphi_{\theta}-\varphi_{0}}{\theta-\theta_{0}} .
$$

First, since $\lambda\left(\theta_{0}\right)=0, \theta \mapsto \varphi_{\theta} \in H^{1}(M)$ is not differentiable at $\theta_{0}$, otherwise differentiating $E L_{\theta}$ with respect to $\theta$ at $\theta_{0}$ would yield a contradiction with (7.19). Hence $\psi_{\theta}$ has no limit in $H^{1}(M)$ for $\theta$ going to $\theta_{0}$. Moreover, $\left(\psi_{\theta}\right)_{\theta}$ is not even bounded for the $H^{1}$ norm up to a subsequence. If we assume the contrary, there exists $\psi_{0} \in H^{1}(M)$ such that $\psi_{\theta}$ converges weakly in $H^{1}(M)$, up to a subsequence, to $\psi_{0}$. Then (7.18) gives

$$
\triangle_{g} \psi_{\theta}+h \psi_{\theta}=f \frac{\varphi_{\theta}^{2^{*}-1}-\varphi_{0}^{2^{*}-1}}{\varphi_{\theta}-\varphi_{0}} \psi_{\theta}+a \theta\left(\frac{1}{\varphi_{\theta}^{2^{*}+1}}-\frac{1}{\varphi_{0}^{2^{*}+1}}\right)\left(\varphi_{\theta}-\varphi_{0}\right)^{-1} \psi_{\theta}+\frac{a}{\varphi_{0}^{2^{*}+1}} .
$$


Now we integrate (17.22) against the eigenvector $u_{0}$ and let $\theta$ go to $\theta_{0}$ : since $\psi_{\theta}$ converges weakly to $\psi_{0}$ we can use Lebesgue's dominated convergence theorem to get

$$
\int_{M}\left\langle\nabla \psi_{0}, \nabla u_{0}\right\rangle d v_{g}+\int_{M}\left[h-\left(2^{*}-1\right) f \varphi_{0}^{2^{*}-2}+\left(2^{*}+1\right) \frac{a \theta_{0}}{\varphi_{0}^{2^{*}+2}}\right] \psi_{0} u_{0}=\int_{M} \frac{a}{\varphi_{0}^{2^{*}+1}} u_{0} d v_{g}>0,
$$

and this yields a contradiction with (7.19). Hence $\left\|\psi_{\theta}\right\|_{H^{1}} \rightarrow+\infty$ as $\theta \rightarrow \theta_{0}$ and this proves (7.20). We now come back to (7.18), pick $\eta=\theta_{0}$, integrate it against $\varphi_{\theta}-\varphi_{0}$ and use once again Lebesgue's dominated convergence theorem. We obtain for any $\theta<\theta_{2}$ :

$$
\begin{aligned}
& \left\|\varphi_{\theta}-\varphi_{0}\right\|_{H_{h}^{1}}^{2} \leq\left(\theta-\theta_{0}\right) \int_{M} \frac{a}{\varphi_{0}^{2^{*}+1}}\left(\varphi_{\theta}-\varphi_{0}\right) d v_{g} \\
& +\left(\left(2^{*}-1\right) \int_{M} f \varphi_{0}^{2^{*}-2} d v_{g}+\theta_{0} \int_{M}\left(2^{*}+1\right) \frac{a}{\varphi_{0}^{2^{*}+2}} d v_{g}+o_{\theta \rightarrow \theta_{0}}(1)\right)\left\|\varphi_{\theta}-\varphi_{0}\right\|_{\infty}^{2} .
\end{aligned}
$$

With (7.20) and since $f>0$ we thus get:

$$
\left\|\psi_{\theta}\right\|_{\infty} \rightarrow+\infty
$$

where $\psi_{\theta}$ is as in (7.21). Define for $\theta \neq \theta_{0}$

$$
\Phi_{\theta}=\frac{\varphi_{\theta}-\varphi_{0}}{\left\|\varphi_{\theta}-\varphi_{0}\right\|_{\infty}} .
$$

Using (7.24) in (7.23) we see that $\Phi_{\theta}$ is $H^{1}$-bounded and then converges weakly, up to a subsequence, to some function $\Phi_{0}$ in $H^{1}(M)$. Since $\left\|\Phi_{\theta}\right\|_{\infty}=1$, using (7.18) and (7.24) we obtain that

$$
\Phi_{\theta} \rightarrow \Phi_{0}=\left\{\begin{array}{r}
u_{0} \text { if } \theta>\theta_{0} \\
-u_{0} \text { if } \theta<\theta_{0}
\end{array}\right.
$$

in $H^{1}(M)$ as $\theta$ goes to $\theta_{0}$, where $u_{0}$ is as in (7.19). By (7.18) and (7.19) we can write:

$$
\begin{aligned}
& \left(\triangle_{g}+h\right)\left(\Phi_{\theta}-\Phi_{0}\right)=f\left(\frac{\varphi_{\theta}^{2^{*}-1}-\varphi_{\theta_{0}}^{2^{*}-1}}{\varphi_{\theta}-\varphi_{0}}\right) \Phi_{\theta}-\left(2^{*}-1\right) f \varphi_{0}^{2^{*}-2} \Phi_{0} \\
& +a\left(\varphi_{\theta}-\varphi_{0}\right)^{-1}\left(\frac{1}{\varphi_{\theta}^{2^{*}+1}}-\frac{1}{\varphi_{\theta_{0}}^{2^{*}+1}}\right) \Phi_{\theta}+\left(2^{*}+1\right) \frac{\theta_{0} a}{\varphi_{0}^{2^{*}+2}} \Phi_{0}+\frac{\theta-\theta_{0}}{\left\|\varphi_{\theta}-\varphi_{0}\right\|_{\infty}} \frac{a}{\varphi_{\theta}^{2^{*}+1}} .
\end{aligned}
$$

Integrating (7.27) against $\Phi_{\theta}-\Phi_{0}$, using (7.24) and using Lebesgue's dominated convergence theorem shows that $\Phi_{\theta}$ converges strongly to $\Phi_{0}$ in $H^{1}(M)$. This gives the following expansion in $H^{1}(M)$ for $\varphi_{\theta}$ as $\theta \rightarrow \theta_{0}$ :

$$
\varphi_{\theta}=\varphi_{0}+\varepsilon_{\theta} u_{0}+o\left(\varepsilon_{\theta}\right),
$$

where we have set $\varepsilon_{\theta}=\operatorname{sgn}\left(\theta-\theta_{0}\right)\left\|\varphi_{\theta}-\varphi_{0}\right\|_{\infty}$. To conclude it remains to integrate (7.19) against $u_{0}$ and use (7.28) and (7.24) to obtain, at the first order in $\varepsilon_{\theta}$ :

$$
\begin{array}{r}
-\left(\int_{M}\left[\left(2^{*}-1\right)\left(2^{*}-2\right) f \varphi_{0}^{2^{*}-3} u_{0}^{3}+\left(2^{*}+1\right)\left(2^{*}+2\right) \frac{\theta_{0} a}{\varphi_{0}^{2^{*}+3}} u_{0}^{3}\right] d v_{g}\right) \varepsilon_{\theta}+o\left(\varepsilon_{\theta}\right) \\
=(1+o(1)) \lambda(\theta) \int_{M} u_{0}^{2} d v_{g} .
\end{array}
$$

As one can check, to obtain (7.29) we used that $u_{\theta} \rightarrow u_{0}$ in $L^{2}(M)$ as $\theta \rightarrow \theta_{0}$. To prove this, first recall the variational characterization of $\lambda(\theta)$ for all $\theta$ as:

$$
\lambda(\theta)=\inf _{\psi \in H^{1}(M),\|\psi\|_{2}=1} \int_{M}\left(|\nabla \psi|^{2}+\left[h-\left(2^{*}-1\right) f \varphi_{\theta}^{2^{*}-2}+\left(2^{*}+1\right) \frac{\theta a}{\varphi_{\theta}^{2^{*}+2}}\right] \psi^{2}\right) d v_{g} .
$$


In particular, this shows that $\lambda: \theta \mapsto \lambda(\theta)$ is upper semi-continuous. Since by its very definition $\varphi_{\theta}$ is stable, $\lambda(\theta)$ is always non negative and there thus holds $\lambda(\theta) \rightarrow 0$ as $\theta \rightarrow \theta_{0}$. Then the convergence of $u_{\theta}$ towards $u_{0}$ in $L^{2}(M)$ holds by standard elliptic theory since $u_{\theta}$ satisfies in addition (7.19) and $\left\|u_{\theta}\right\|_{\infty}=1$ for all $0<\theta<\theta_{2}$. Since we assumed $f>0$ we see with (7.29) that $\lambda$ changes sign at $\theta_{0}$. By assumption $\lambda(\theta)$ is always non negative: we have a contradiction and $D^{2} I_{\theta}\left(\varphi_{\theta}\right)$ is definite positive for all $0<\theta<\theta_{2}$. Application of Theorem 5.1 is then straightforward and gives a second solution of $\left[E L_{\theta}\right]$ when $0<\theta<\theta_{2}$. Letting $\theta_{\star}=\theta_{2}$, we have thus shown that, if $f>0,(E L)$ has at least two solutions for $\theta<\theta_{\star}$ and none for $\theta>\theta_{\star}$. Now we show that in the limit case $\theta=\theta_{\star}$ there is a unique solution. Its existence is obtained with a stability argument and its uniqueness is a consequence of the non coercivity of $D^{2} I_{\theta_{\star}}\left(\varphi_{\theta_{\star}}\right)$, where $I_{\theta_{\star}}$ is as in (7.15). First, a solution exists for $\theta=\theta_{\star}$. Indeed, let $\left(\theta_{k}\right)_{k}, 0<\theta_{k}<\theta_{\star}$ be a sequence converging to $\theta_{\star}$ (which is finite since $f>0$ ). Then by Theorem 2.1 applied with $q_{k}=2^{*}$ and $a_{k}=\theta_{k} a$ in the case $f>0$, the sequence $\varphi_{\theta_{k}}$ converges in $C^{1, \alpha}(M)$ to some smooth positive function $\varphi_{\theta_{\star}}$ that solves $\left.E L_{\theta}\right]$ with $\theta=\theta_{\star}$. To prove uniqueness, we first show that the minimal solution $\varphi_{\theta_{\star}}$ is not strictly stable. For the sake of simplicity, we let $\varphi_{\star}=\varphi_{\theta_{\star}}$. We proceed by contradiction and assume that $\varphi_{\star}$ is strictly stable: that is, we assume that

$$
\lambda\left(\theta_{\star}\right)>0,
$$

where $\lambda\left(\theta_{\star}\right)$ is the first eigenvalue of the linearization of $\left.E L_{\theta}\right]$ for $\theta=\theta_{\star}$ at $\varphi_{\star}$. Let $0<\alpha<1$ : since we assumed that $\triangle_{g}+h$ is coercive it is, by standard elliptic theory, an isomorphism between $C^{k+2, \alpha}(M)$ and $C^{k, \alpha}(M)$ for any integer $k \geq 0$. Let $\delta=\frac{1}{2} \inf _{M} \varphi_{\star}>0, B\left(\varphi_{\star}, \delta\right)=$ $\left\{v \in C^{2, \alpha}(M)\right.$ s.t. $\left.\left\|v-\varphi_{\star}\right\|_{C^{2, \alpha}} \leq \delta\right\}$ and $\Omega=B\left(\varphi_{\star}, \delta\right) \times \mathbb{R}$. The following mapping is thus well-defined:

$$
F:\left\{\begin{aligned}
\Omega & \longrightarrow C^{2, \alpha}(M) \\
(u, \theta) & \longmapsto u-\left(\triangle_{g}+h\right)^{-1}\left(f u^{2^{*}-1}+\frac{\theta a}{u^{2^{*}+1}}\right) .
\end{aligned}\right.
$$

It is easily seen that $F$ is $C^{1}$ in $\Omega$ and that there holds, for all $(u, \theta) \in \Omega$ and for all $v \in C^{2, \alpha}(M)$ :

$$
\frac{\partial F}{\partial u}(u, \theta)(v)=v-\left(\triangle_{g}+h\right)^{-1}\left(\left(2^{*}-1\right) f u^{2^{*}-2} v-\left(2^{*}+1\right) \frac{\theta a}{u^{2^{*}+2}} v\right) .
$$

We claim that $\frac{\partial F}{\partial u}\left(\varphi_{\star}, \theta_{\star}\right)$ is an isomorphism. Indeed, let $w \in C^{2, \alpha}(M)$. Finding $v \in C^{2, \alpha}(M)$ such that

$$
\frac{\partial F}{\partial u}\left(\varphi_{\star}, \theta_{\star}\right)(v)=w
$$

is equivalent to solve

$$
\triangle_{g} v+\left[h-\left(2^{*}-1\right) f \varphi_{\star}^{2^{*}-2}+\left(2^{*}+1\right) \frac{\theta_{\star} a}{\varphi_{\star}^{2^{*}+2}}\right] v=\left(\triangle_{g}+h\right) w .
$$

By (7.30) the left-hand side is a coercive linear operator. Since $\triangle_{g} w+h w \in C^{0, \alpha}(M)$ there exists, by standard elliptic theory, a unique $v \in C^{2, \alpha}(M)$ satisfying (7.33), and hence (7.32). As one can check, $F\left(\varphi_{\star}, \theta_{\star}\right)=0$ and more generally, for any $(u, \theta) \in \Omega, u$ solves $\left.E L_{\theta}\right]$ if and only if $F(u, \theta)=0$. With (7.32) the implicit function theorem applies to $F$ at $\left(\varphi_{\star}, \theta_{\star}\right)$ and shows that the set of solutions of the equation $F(u, \theta)=0$ is locally a path in $B\left(\varphi_{\star}, \delta\right)$ parameterized by $\theta \in\left(\theta_{\star}-\varepsilon, \theta_{\star}+\varepsilon\right)$ for some positive $\varepsilon$. But this is impossible since no solutions of $E L_{\theta}$ exist if $\theta>\theta_{\star}$.

Hence $\lambda\left(\theta_{\star}\right)=0$ and there exists a positive $\eta \in C^{\infty}(M)$ such that

$$
\triangle_{g} \eta+\left[h-\left(2^{*}-1\right) f \varphi_{\star}^{2^{*}-2}+\left(2^{*}+1\right) \frac{\theta_{\star} a}{\varphi_{\star}^{2^{*}+2}}\right] \eta=0 .
$$


The uniqueness of solutions for $\theta=\theta_{\star}$ then follows as in Ma-Wei [18. If there exists a solution $v$ different from $\varphi_{\star}$, by minimality of $\varphi_{\star}$ there would hold $v>\varphi_{\star}$. Since $f>0$ in $M$ and $a \geq 0$ in $M$, for any $x \in M$ the functions $u \in \mathbb{R} \mapsto f(x) u^{2^{*}-1}$ and $u \in \mathbb{R} \mapsto \frac{\theta_{\star} a(x)}{u^{2^{*}+1}}$ are respectively strictly convex and convex. We therefore obtain:

$$
\triangle_{g}\left(v-\varphi_{\star}\right)+\left[h-\left(2^{*}-1\right) f \varphi_{\star}^{2^{*}-2}+\left(2^{*}+1\right) \frac{\theta_{\star} a}{\varphi_{\star}^{2^{*}+2}}\right]\left(v-\varphi_{\star}\right)>0
$$

and integrating (7.35) against $\eta$ and using (7.34) gives a contradiction. This concludes the proof of Theorem 1.1.

\section{REFERENCES}

[1] P. T. Allen, A. Clausen, and J. Isenberg. Near-constant mean curvature solutions of the Einstein constraint equations with non-negative Yamabe metrics. Classical Quantum Gravity, 25(7):075009, 15, 2008.

[2] T. Aubin. Nonlinear analysis on manifolds. Monge-Ampère equations, volume 252 of Grundlehren der Mathematischen Wissenschaften [Fundamental Principles of Mathematical Sciences]. Springer-Verlag, New York, 1982 .

[3] R. Bartnik and J. Isenberg. The constraint equations. In The Einstein equations and the large scale behavior of gravitational fields, pages 1-38. Birkhäuser, Basel, 2004.

[4] R. Beig, P. T. Chruściel, and R. Schoen. KIDs are non-generic. Ann. Henri Poincaré, 6(1):155-194, 2005.

[5] L. A. Caffarelli, B. Gidas, and J. Spruck. Asymptotic symmetry and local behavior of semilinear elliptic equations with critical Sobolev growth. Comm. Pure Appl. Math., 42(3):271-297, 1989.

[6] Y. Choquet-Bruhat, J. Isenberg, and D. Pollack. Applications of theorems of jean leray to the Einstein-scalar field equations. J. Fixed Point Theory Appl., 1(1):31-46, 2007.

[7] Y. Choquet-Bruhat, J. Isenberg, and D. Pollack. The constraint equations for the Einstein-scalar field system on compact manifolds. Classical Quantum Gravity, 24(4):809-828, 2007.

[8] M. Dahl, R. Gicquaud, and E. Humbert. A limit equation associated to the solvability of the vacuum Einstein constraint equations using the conformal method. Duke Math. J. To appear.

[9] O. Druet. Compactness for Yamabe metrics in low dimensions. Int. Math. Res. Not., (23):1143-1191, 2004.

[10] O. Druet and E. Hebey. Stability and instability for Einstein-scalar field Lichnerowicz equations on compact Riemannian manifolds. Math. Z., 263(1):33-67, 2009

[11] Y. Fourès-Bruhat. Théorème d'existence pour certains systèmes d'équations aux dérivées partielles non linéaires. Acta Math., 88:141-225, 1952.

[12] E. Hebey, F. Pacard, and D. Pollack. A variational analysis of Einstein-scalar field Lichnerowicz equations on compact Riemannian manifolds. Comm. Math. Phys., 278(1):117-132, 2008.

[13] E. Hebey and G. Veronelli. The lichnerowicz equation in the closed case of the einstein-maxwell theory. Trans. Amer. Math. Soc., 2011. Accepted for publication.

[14] M. Holst, G. Nagy, and G. Tsogtgerel. Rough solutions of the Einstein constraints on closed manifolds without near-CMC conditions. Comm. Math. Phys., 288(2):547-613, 2009.

[15] J. Isenberg. Constant mean curvature solutions of the Einstein constraint equations on closed manifolds. Classical Quantum Gravity, 12(9):2249-2274, 1995.

[16] Y. Li and M. Zhu. Yamabe type equations on three-dimensional Riemannian manifolds. Commun. Contemp. Math., 1(1):1-50, 1999.

[17] A. Lichnerowicz. L'intégration des équations de la gravitation relativiste et le problème des $n$ corps. J. Math. Pures Appl. (9), 23:37-63, 1944.

[18] L. Ma and J. Wei. Stability and multiple solutions to einstein-scalar field lichnerowicz equation on manifolds. J. Math. Pures Appl., 2012. http://dx.doi.org/10.1016/j.matpur.2012.06.009.

[19] D. Maxwell. A class of solutions of the vacuum Einstein constraint equations with freely specified mean curvature. Math. Res. Lett., 16(4):627-645, 2009.

[20] Q. A. Ngô and X. Xu. Existence results for the Einstein-scalar field Lichnerowicz equations on compact Riemannian manifolds. Adv. Math., 230(4-6):2378-2415, 2012.

[21] B. Premoselli. The einstein-scalar field constraint system in the positive case. Comm. Math. Phys. To appear.

[22] P. H. Rabinowitz. Minimax methods in critical point theory with applications to differential equations, volume 65 of CBMS Regional Conference Series in Mathematics. Published for the Conference Board of the Mathematical Sciences, Washington, DC, 1986.

[23] F. Robert. Existence et asymptotiques optimales des fonctions de green des opérateurs elliptiques d'ordre deux. 2009. Personal notes. 
[24] D. H. Sattinger. Monotone methods in nonlinear elliptic and parabolic boundary value problems. Indiana Univ. Math. J., 21:979-1000, 1971/72.

[25] R. M. Schoen. Lecture notes from courses at stanford. 1988. Written by D. Pollack, preprint.

[26] R. M. Schoen. On the number of constant scalar curvature metrics in a conformal class. In Differential geometry, volume 52 of Pitman Monogr. Surveys Pure Appl. Math., pages 311-320. Longman Sci. Tech., Harlow, 1991.

[27] M. Struwe. Variational methods, volume 34 of Ergebnisse der Mathematik und ihrer Grenzgebiete. 3. Folge. A Series of Modern Surveys in Mathematics [Results in Mathematics and Related Areas. 3rd Series. A Series of Modern Surveys in Mathematics]. Springer-Verlag, Berlin, fourth edition, 2008. Applications to nonlinear partial differential equations and Hamiltonian systems.

Laboratoire de Mathématiques - AGM, Université de Cergy-Pontoise, 2 Avenue Adolphe Chauvin 95302 Cergy-Pontoise Cedex and

Laboratoire de Mathématiques - UMpa, Ecole Normale Supérieure de Lyon (site sciences), 46 allée d'Italie, 69364 Lyon Cedex 07.

E-mail address: bruno.premoselli@u-cergy.fr 